\title{
SENSORIMOTOR CORTICAL INFLUENCES ON CUNEATE NUCLEUS RHYTHMIC ACTIVITY IN THE ANESTHETIZED CAT
}

\author{
J. MARIÑO, A. CANEDO* and J. AGUILAR \\ Department of Physiology, Faculty of Medicine, Laboratory of Neuroscience and Neuronal Computation, \\ associated with the Cajal Institute (CSIC), Santiago de Compostela, Spain
}

\begin{abstract}
This work aimed to study whether the sensorimotor cerebral cortex spreads down its rhythmic patterns of activity to the dorsal column nuclei. Extracellular and intracellular recordings were obtained from the cuneate nucleus of chloralose-anesthetized cats. From a total of 140 neurons tested (106 cuneolemniscal), 72 showed spontaneous rhythmic activity within the slow $(<1 \mathrm{~Hz}), \delta$ $(1-4 \mathrm{~Hz})$, spindle $(5-15 \mathrm{~Hz})$ and higher frequencies, with seven cells having the $\delta$ rhythm coupled to slow oscillations. The spindle activity recorded in the cuneate was tightly coupled to the thalamo-cortico-thalamic spindle rhythmicity. Bilateral or contralateral removal of the frontoparietal cortex abolished the cuneate slow and spindle oscillations. Oscillatory paroxysmal activity generated by fast electrical stimulation $(50-100 \mathrm{~Hz} / 1-2 \mathrm{~s})$ of the sensorimotor cortex induced burst firing synchronized with the paroxysmal cortical "spike" on all the non-lemniscal neurons, and inhibitory responses also coincident with the cortical paroxysmal "spike" in the majority (71\%) of the cuneolemniscal cells. The remaining lemniscal-projecting neurons showed bursting activity (11\%) or sequences of excitation-inhibition (18\%) also time-locked to the cortical paroxysmal "spike". Additionally, the cerebral cortex induced coherent oscillatory activity between thalamic ventroposterolateral and cuneate neurons. Electrolytic lesion of the pyramidal tract abolished the cortically induced effects on the contralateral cuneate nucleus, as well as on the ipsilateral medial lemniscus.

The results demonstrate that the sensorimotor cortex imposes its rhythmic patterns on the cuneate nucleus through the pyramidal tract, and that the corticocuneate network can generate normal and abnormal patterns of synchronized activity, such as $\delta$ waves, spindles and spike-and-wave complexes. The cuneate neurons, however, are able to generate oscillatory activity above $1 \mathrm{~Hz}$ in the absence of cortical input, which implies that the cerebral cortex probably imposes its rhythmicity on the cuneate by matching the intrinsic preferred oscillatory frequency of cuneate neurons. (C) 1999 IBRO. Published by Elsevier Science Ltd.
\end{abstract}

Key words: sensorimotor cortex, cuneate nucleus, synchronized activity, cat.

The neocortex and the thalamus form an oscillatory network modulated by ascending excitatory and inhibitory influences arising mostly in the brainstem. The activity in the network varies according to the behavioral state: during quiet sleep and drowsiness the cortical and thalamic neurons show synchronized rhythmic bursting activity at low frequencies $(0.1-15 \mathrm{~Hz})$, while during wakefulness and rapid eye movement sleep the frequency of the synchronized oscillations increases to $20-80 \mathrm{~Hz}$ (mainly $30-40 \mathrm{~Hz}$ ) with the neurons showing tonic, sustained discharges. ${ }^{21,43,48}$ These two neuronal modes of activity (bursting and tonic) are recognized to serve two basic functions: the bursting mode implies a functional disconnection from the outside world, since it is produced through intrinsic neuronal membrane properties which do not reflect the attributes of the sensory signals; the tonic or transfer mode allows the faithful relay of sensory inputs. ${ }^{50}$

In general, prethalamic structures such as the dorsal column nuclei (DCN; cuneatus and gracilis) are not considered to play a major role in the self-sustained patterns of operation of the thalamo-cortico-thalamic networks during sleep and anesthesia. This is probably due to the fact that descending connections from the specific somatosensory

\footnotetext{
*To whom correspondence should be addressed. Tel.: +34-981-582658 ext. 12292; fax: +34-981-574145.

E-mail address: fsancala@usc.es (A. Canedo)

Abbreviations: CL, cuneolemniscal neuron; DCN, dorsal column nuclei; ECoG, electrocorticogram; EPSP, excitatory postsynaptic potential; IPSP, inhibitory postsynaptic potential; ML, medial lemniscus; nCL, non-cuneolemniscal neuron; PT, pyramidal tract; VPL, ventroposterolateral thalamic nucleus.
}

thalamus to the DCN have not been described. The DCN, however, supply afferents to the ventroposterolateral thalamic nucleus (VPL) and receive abundant corticofugal input through the pyramidal tract (PT), ${ }^{29}$ thus potentially affecting the thalamocortical activity whether during bursting, tonic or both modes of operation, probably under the influence of the cerebral cortex.

It has recently been demonstrated that the existence of lowfrequency rhythmic activity (mostly slow, $<1 \mathrm{~Hz}$, and $\delta, 1-$ $4 \mathrm{~Hz}$ ) in the cuneate nucleus is tightly coupled to the same oscillating patterns of VPL neurons. ${ }^{28}$ It was also shown that the cuneate neurons display tonic and bursting activity which, as in the thalamus and the cortex, appears to depend partially on intrinsic membrane properties. ${ }^{7,31}$ It was suggested that, while the cortically originated slow oscillation ${ }^{47,51,52}$ is probably transmitted through corticocuneate anatomical pathways, the $\delta$ cuneate rhythmicity might be intrinsically generated, ${ }^{7,28}$ but whether the cuneate oscillatory activity, in the anesthetized animal, is imposed by the cerebral cortex remains to be demonstrated. It was shown that near-simultaneous spindle waves appear in different thalamic sites of anesthetized cats ${ }^{13}$ and that this large-scale synchrony is disrupted by removal of the cerebral cortex, ${ }^{12}$ which indicates that intrathalamic mechanisms are insufficient to account for the large-scale synchrony of oscillations in vivo and emphasizes the role of corticothalamic feedback in large-scale synchrony. ${ }^{15}$ This also poses the question of whether the cerebral cortex spreads the thalamocortical spindle rhythmicity on prethalamic structures such as the DCN.

According to the above, the present work aimed to study whether the cerebral cortical rhythmic activity is reflected in 
the cuneate nucleus and, if so, through which anatomical route. This question is of undoubted functional importance, since if the neocortex imposes its rhythmic patterns of activity on the subcortical structures receiving direct corticofugal input, then the cortex may coherently induce oscillatory activity on these structures, thus deafferenting itself during the states of sleep and deep anesthesia. A second concern of the study was to ascertain whether the sensorimotor corticofugal fibers induced coherent oscillatory activity along the somatosensory ascending pathway, in the cuneate nucleus and VPL.

To deal with these questions, experiments were conducted on anesthetized cats, since the central rhythmic neuronal activity that characterizes sleep and drowsiness is reasonably well replicated in the anesthetized preparation, ${ }^{14}$ and the longaxoned neocortical neurons show oscillatory activity. ${ }^{30}$ The activity of cuneolemniscal cells (CLs) and non-cuneolemniscal cells (nCLs) was recorded extra- and intracellularly before and after lesion of the PT or bilateral removal of the frontoparietal cortex to ascertain whether the cortex influences the cuneate through the PT, and which cuneate oscillations, if any, are preserved in the absence of corticocuneate input. Simultaneous recordings were also obtained from the VPL and the contralateral cuneate nucleus to study whether the cortex induces coherent oscillatory activity on both structures. Finally, the sensorimotor cerebral cortex was electrically stimulated at fast rates to synchronize the corticofugal neurons and thus maximize the probability of recording from cuneate cells receiving cortical input.

Preliminary results have been reported in abstract form. ${ }^{6,26,27}$

\section{EXPERIMENTAL PROCEDURES}

\section{General}

All experiments conformed to local and international guidelines on the ethical use of animals and all efforts were made to minimize the number of animals used. A total of 57 cats of either sex $(2.5-4.2 \mathrm{~kg})$ were anesthetized ( $\alpha$-chloralose, $60 \mathrm{mg} / \mathrm{kg}$, i.p.), paralysed (Pavulon, $1 \mathrm{mg} / \mathrm{kg} / \mathrm{h}$, i.v.) and artificially respired at $15-25$ strokes $/ \mathrm{min}$, with $20-40 \mathrm{ml} /$ stroke according to body weight. Additional doses of anesthesia were administered when necessary. The depth of anesthesia was assessed by monitoring the heart rate and the electrocorticogram (ECoG; digitally filtered at a frequency band-pass of 1-200 Hz, or 10$1000 \mathrm{~Hz}$ when analysing single spikes), and by observing the state of the pupil. Changes of heart rate (maintained in the range of 90120 beats/min) or in the pattern of the ECoG, and dilated pupils, or pupils reacting rapidly to electrical stimuli were considered to reflect inadequate anesthesia, and supplementary doses of $30-40 \mathrm{mg} / \mathrm{kg}$ of $\alpha$-chloralose were injected every $4-6 \mathrm{~h}$, as required. Rectal temperature was maintained at $37.5 \pm 0.5^{\circ} \mathrm{C}$ by an abdominal heating pad under servocontrol. Tracheal and venous cannulae were inserted, and the animal was positioned in a stereotaxic frame with the head flexed by about $30-40^{\circ}$ to allow easy access to the dorsal medulla. The animals were suspended with clamps attached to the thoracic and lumbar vertebrae. The dorsal medulla was exposed to insert recording electrodes into the middle main cuneate nucleus from the level of the obex to $4 \mathrm{~mm}$ caudal to it. To minimize pulsatile movements, a bilateral pneumothorax was routinely carried out, and a $4 \%$ agar solution in $0.9 \%$ saline at $38^{\circ} \mathrm{C}$ was poured over the exposed medulla to a height of about $20 \mathrm{~mm}$.

\section{Stimulation}

To identify antidromically CLs, a bipolar stimulating electrode (0.5 mm intertip space, $40-50 \mu \mathrm{m}$ diameter and insulated except at the tip) was introduced through a craniotomy into the contralateral medial lemniscus (ML) at Horsley-Clarke stereotaxic coordinates A 2 , L 4.5, H -5 . Recording of evoked potentials and multiunitary responses to mechanical and electrical stimulation of the contralateral distal forelimb assured correct placement. ${ }^{7}$ CLs were antidromically identified according to standard criteria including, in all cases, the collision test (Fig. 1). A set of six bipolar stimulating electrodes was mounted in a tower and lowered to $1-1.5 \mathrm{~mm}$ deep in the pericruciate cortex to stimulate corticocuneate neurons. These electrodes were disposed in two rows aligned mediolaterally in the anterior and posterior gyrus sigmoideus, so that the primary motor and primary somatosensory cortices could be stimulated (see Fig. 5A). Iterative electrical stimulation of the sensorimotor cortex (trains of 1-2 s duration at 50 $100 \mathrm{~Hz}$ ) was used to induce cortical synchronization. Cathodal shocks of $0.05 \mathrm{~ms}$ duration and with intensities of up to $0.5 \mathrm{~mA}$ were applied to the ML. Lower stimulating intensities of up to $0.2 \mathrm{~mA}$ were applied to the cortex to avoid spread of current to the corona radiata. Rectangular pulses were applied between both terminals of each bipolar electrode. Electrical stimuli were isolated from the ground providing constant current to the preparation by using a transistorized optically coupled unit (Grass PSIU6, Quincy, MA) placed as close to the preparation as possible, and connected in series with the output of a dual pulse digital stimulator (Grass S8800) and the stimulating electrodes.

Peripheral qualitative stimulation consisted of moving joints passively for proprioceptors, and brushing, touching or pressing skin and deeper tissues for other receptors. A narrow jet of compressed air, as well as light brushing with a vibrissal hair held in a small chuck, were used to stimulate hair receptors.

\section{Mass and multiunitary recording}

In all the animals, one bipolar concentric electrode (resistance about $50 \mathrm{k} \Omega$ ) was placed in the lateral tip of the cruciate sulcus at a depth of $1 \mathrm{~mm}$ to obtain differential recordings between the inner (active) and the ring (reference) leads, separated by $500 \mu \mathrm{m}$ (ECoG).

Extracellular recordings (tungsten electrodes, 2-12 M $\Omega$ resistance) were performed on 21 animals. Single and multiunitary recordings were obtained from subcortical structures of 16 animals in different combinations, with eight of these animals having the frontoparietal cortex removed to study the cuneate rhythmic activity in the absence of cortical influences. Data were obtained by unit and field potential recordings from the VPL, the middle main cuneate and the ML, to study the putative effects imposed by the sensorimotor cortex on these structures. In five animals with the cortex intact, multiunitary recordings were obtained simultaneously from VPL and contralateral cuneate neurons with overlapping cutaneous receptive fields. Three other animals were used to record bilaterally from both MLs to study the effects induced by patterned stimuli applied to the sensorimotor cortex of one hemisphere. In five animals, both ipsilateral lemniscal and contralateral cuneate mass recordings were accomplished to study the effects of sensorimotor cortical stimulation before and after lesion of the PT. The PT was ventrally approached at mid-olivary level and a set of two bipolar steel electrodes was mounted in a tower, aligned mediolaterally over the PT under visual guidance and left in place. Anodal direct currents $(0.5-1 \mathrm{~mA})$ were passed through both electrodes until the synchronous multiunit cuneate discharges imposed by the cortical patterned stimuli disappeared from the recording.

\section{Intracellular recording}

Intracellular recording was performed on 33 animals different from those used for extracellular recording. In three animals, the frontoparietal cortex was bilaterally removed to study whether cuneate cells presented rhythmic activity in the absence of corticocuneate input. Only cuneate neurons responding to cortical stimulation were selected from the animals with intact cerebral cortices. Sharp electrodes were used to obtain intracellular recordings from cuneate cells. The resistance of the electrodes measured in the neural tissue ranged from 30 to $50 \mathrm{M} \Omega$ with the micropipettes filled with potassium acetate $(2.5 \mathrm{M})$. Before penetration, the electrode was positioned over the main cuneate nucleus using a motorized driver (Narishige ME-71, Japan), the tissue was then covered with warm agar and, when solidified, the electrode was introduced and the search for cuneate cells began. Electrode resistance was continuously checked by observing the change in voltage produced by current pulses using an axoclamp-2B intracellular amplifier in the bridge mode (Axon Instruments, Foster City, CA). The bridge was balanced and the capacitance neutralization adjusted to give the fastest step response to a current pulse. Electrical stimulation was applied to the ML and to the cortex while the electrode was advanced in 1- to $2-\mu \mathrm{m}$ steps. Since there is a 


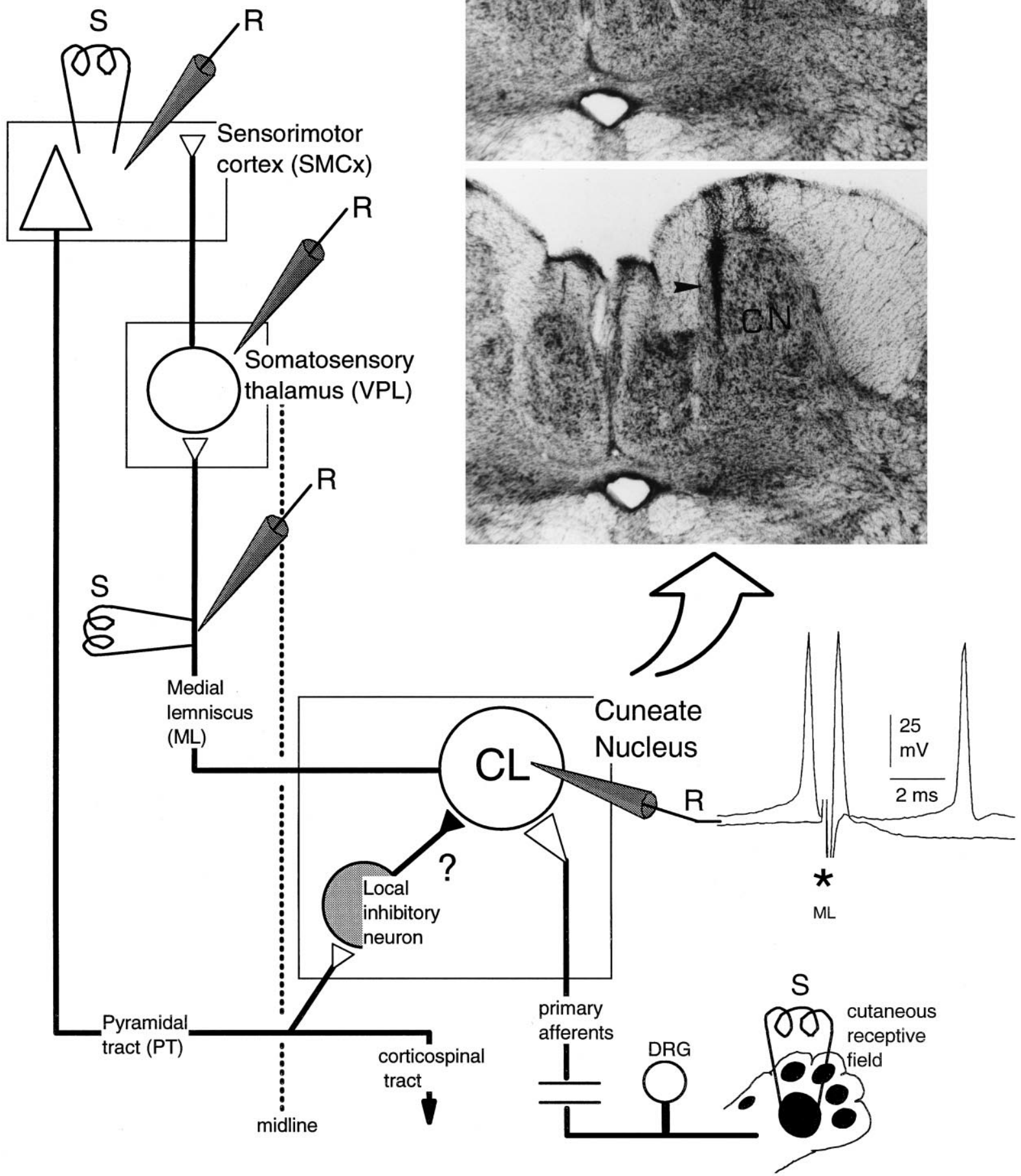

Fig. 1. General experimental arrangement. The inset on the right top shows two electrode tracks through the middle main cuneate nucleus (signaled by white arrowheads). The intracellular recording electrode schematized within a CL points to two superimposed records in one of which a collision between one orthodromic and one antidromic spike occurred. ML stimulus artifacts are marked by asterisks. R, recording; S, stimulation; DRG, dorsal root ganglia. 
powerful cortical influence (mainly from area 4) to the tegmental region ventrolaterally located to the cuneate, ${ }^{10}$ the recordings were restricted to a region located between 0.7 and $1.5 \mathrm{~mm}$ laterally from the midline, and between 0.5 and $1.6 \mathrm{~mm}$ deep from the surface. Stimulation of the excitatory peripheral receptive fields (limited to the homolateral forelimb), as well as reconstruction of the electrode tracks, revealed that the recordings were obtained from the middle main cuneate nucleus (see inset in Fig. 1). Finally, intracellular recordings were obtained from VPL neurons while simultaneously recording the mass discharge in the cuneate of three non-lesioned animals.

\section{Data analysis}

The extra- and intracellular signals were digitized at $22-44 \mathrm{kHz}$ through an eight-channel digitizing unit (Neurodata DR-890) and stored unfiltered on videotape for off-line computer analysis. Extracellular spontaneous activity was sampled over periods of 5-30 min. In lesioned animals, spontaneous activity was sampled before PT or frontoparietal cortical lesions, throughout a 5- to 10-min period immediately after the lesions and then at longer intervals spaced throughout the recording. Single action potentials from multiunitary recordings were played back and continuously monitored to avoid processing spikes originating in other units of the recording with similar shapes and waveforms. Two voltage windows were cascaded for spike discrimination, one above and the other below baseline, whenever various spikes had similar waveforms. The acceptance pulse of the second window was led to a computer through a 1401-plus interface, stored and processed using spike2 software (CED, Cambridge, U.K.). The extracellular recordings were low-pass filtered at $100 \mathrm{~Hz}$ to eliminate slow waves that could interfere with the analysis. Auto- and crosscorrelograms of single spikes were computed for periods of 2$10 \mathrm{~min}$ of stable activity to reveal the frequency of oscillations and to determine the degree of synchrony between neuronal discharges, respectively. Auto- and cross-correlograms were considered rhythmic when displaying two or more distinguishable peaks. ${ }^{14}$ The strength of the oscillation was assessed by counting the secondary peaks in the auto-correlations according to the method described by Amzica and Steriade. ${ }^{1}$ The spikes of different cells were considered synchronous when they occurred within 1-5 ms of each other.

The intracellular signals were played back, digitized at $10-20 \mathrm{kHz}$ (Digidata 1200) and analysed using AxoScope software (Axon Instruments)

\section{Histology}

At the end of each experiment, positive current (100 $\mu \mathrm{A}$ for $20 \mathrm{~s})$ was passed through the stimulating electrodes to mark their tip positions by electrolytic lesions. The animal was perfused with normal saline followed by $4 \%$ paraformaldehyde. The neural tissues of interest were removed and postfixed. Transverse $60-\mu \mathrm{m}$ frozen sections were cut, serially mounted, stained, and the locations of recording and stimulating sites determined. Reconstruction of electrode tracks revealed that the recordings were obtained from the middle main cuneate nucleus (see inset in Fig. 1).

\section{RESULTS}

\section{General}

Intracellular $(n=52)$ and extracellular single recordings $(n=54)$ were obtained from CLs identified antidromically and from 88 nCLs (40 recorded intracellularly and 48 recorded extracellularly) in cats with intact cortex. Five additional cells (three CLs, two nCLs) were recorded intracellularly from three cats with the frontoparietal cortex bilaterally removed. Five VPL neurons that responded to movement of hairs (two cells) or to light touch (three cells) in the contralateral distal forelimb were also recorded intracellularly simultaneously to the mass discharge in the cuneate. This was done to determine whether there was any temporal correlation between the membrane fluctuations of the thalamic cells and the cuneate gross discharge during spindle oscillations.
Identification of cuneolemniscal and non-cuneolemniscal cells

Strong cathodal shocks were applied to the ML while gradually increasing the intensity and frequency of stimulation to separate CLs from nCLs. To distinguish corticofugal fibers entering the ventral part of the nucleus from cuneate neurons, high-frequency stimulation was applied to the sensorimotor cerebral cortex. The neurons showed cortically evoked synaptic responses while the presumed cortical fibers did not. The example shown in Fig. 2 illustrates this point. The first trace in Fig. 2A shows a CL that responded to ML stimulation generating an antidromic action potential followed by a trans-synaptic excitatory postsynaptic potential (EPSP) crowned by a doublet of spikes. The cell responded to $100-\mathrm{Hz}$ iterative stimulation of the primary somatosensory cortex with a first burst of four spikes followed by compound EPSPs, occasionally leading to action potentials (Fig. 2A, lower trace). In contrast, the presumed cortical fibers did not show synaptic potentials in response to cortical stimulation either with increasing stimulating frequencies (Fig. 2B; $100 \mathrm{~Hz}$ in the first trace and $200 \mathrm{~Hz}$ in the second) or with increasing stimulating intensities (Fig. 2B, lower trace).

\section{Cuneate rhythmicity}

The use of single spikes of cuneate neurons to compile autocorrelograms demonstrated that both nCLs (Fig. 3A) and CLs (Fig. 3B) showed slow, $\delta$, spindle and higher $(>15 \mathrm{~Hz})$ rhythmic activities at rest, with the CLs having a clear tendency to discharge in bursts of two to five spikes (inset in Fig. 3B2). The CLs discharged preferentially within the $\delta$ band, while the nCLs tended to fire at higher frequencies presenting a more tonic behavior, as was reported for gracile neurons in barbiturate- and urethane-anesthetized rats. ${ }^{32}$ Furthermore, some of the rhythmic cuneate neurons showed the $\delta$ activity grouped into slow rhythms (Table 1, Fig. 3A1).

\section{Spontaneous synchronization}

Unitary extracellular single spikes, recorded through the ECoG electrode in the sensorimotor cortex of three animals, presented rhythmic activity tightly coupled to single fiber activity in the ipsilateral ML (Fig. 4).

\section{Simultaneous recordings in the ventroposterolateral thalamic nucleus and cuneate}

In five animals, simultaneous extracellular recordings were obtained from VPL and cuneate neurons with overlapping peripheral cutaneous receptive fields. From a total sample of 25 recorded pairs, 12 showed spontaneous coupled activity within the range of slow and $\delta$ frequencies, as reported previously. ${ }^{28}$ The remaining 13 pairs showed uncoupled rhythmic activities, but the majority (eight of 13) could be induced to discharge synchronously when stimulating either the motor or the somatosensory cortex at low frequency $(0.5-$ $1 \mathrm{~Hz}$; data not shown), as demonstrated previously for thalamic cells. ${ }^{14}$ Five VPL neurons recorded intracellularly showed clear membrane potential fluctuations tightly coupled to the electrocorticographic spindles, which were also reflected in the cuneate gross discharges (Fig. 5). Thus, the cerebral cortex spreads down to the cuneate nucleus the slow, $\delta$ and spindle oscillations. Whether the cortical spindles are 


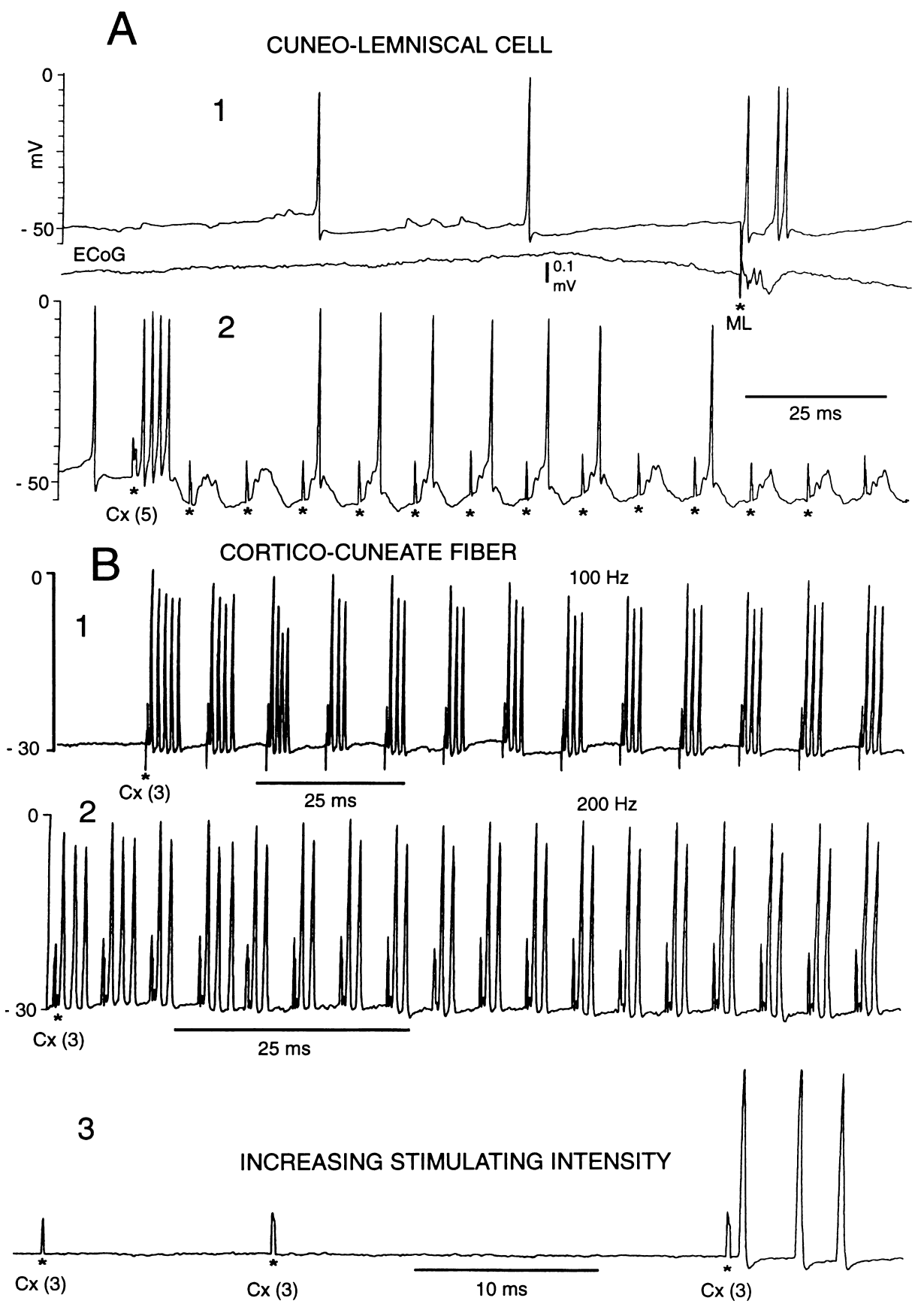

Fig. 2. Distinction between corticocuneate fibers and cuneate neurons. (A) High-frequency stimulation of the somatosensory cortex $[\mathrm{Cx}(5)$ in Fig. $5 \mathrm{~A}]$ induced postsynaptic potentials (A2) in a CL that responded to ML stimulation generating a late burst of two to three spikes following the antidromic response (A1). (B) A corticocuneate fiber showing the absence of postsynaptic potentials when applying high-frequency stimulation (B1, B2) or a gradual increase in stimulating strength (B3) to the motor cortex [Cx(3) in Fig. 5A]. The lower trace in A1 is the ECoG.

reflected on a particular subset of neurons remains to be demonstrated since, in this series, mass recordings were obtained from the cuneate.

Cortical synchronization induced coupled activity in subcortical structures

Trains of fast stimuli applied to the sensorimotor cortex (0.05 ms pulse duration, $50-100 \mathrm{~Hz} / 1-2 \mathrm{~s})$ induced selfsustained paroxysms. The onset of the paroxysmal activity lagged the end of the stimulating tetani by $150-600 \mathrm{~ms}$ when recorded through an electrode in a transition zone between motor and somatosensory cortices (R1 in Fig. 6A), and by $200-1100 \mathrm{~ms}$ when recorded simultaneously from a different electrode placed $7 \mathrm{~mm}$ apart, in the primary somatosensory cortex (R2 in Fig. 6A). The duration of the paroxysmal discharges ranged from 5 to $15 \mathrm{~s}$ and the frequency of the oscillations decreased from $7-20 \mathrm{~Hz}$ at the onset of the paroxysms to $0.5-6 \mathrm{~Hz}$ at the end, but within these ranges duration and frequency varied between trials and among different animals. In general terms, the induced paroxysmal oscillating activity was initiated with fast runs and gradually increased in amplitude and slowed in frequency until reaching spike-and-wave complexes at $0.5-6 \mathrm{~Hz}$. The epoch with 


\section{A Non-Lemniscal Neurons}
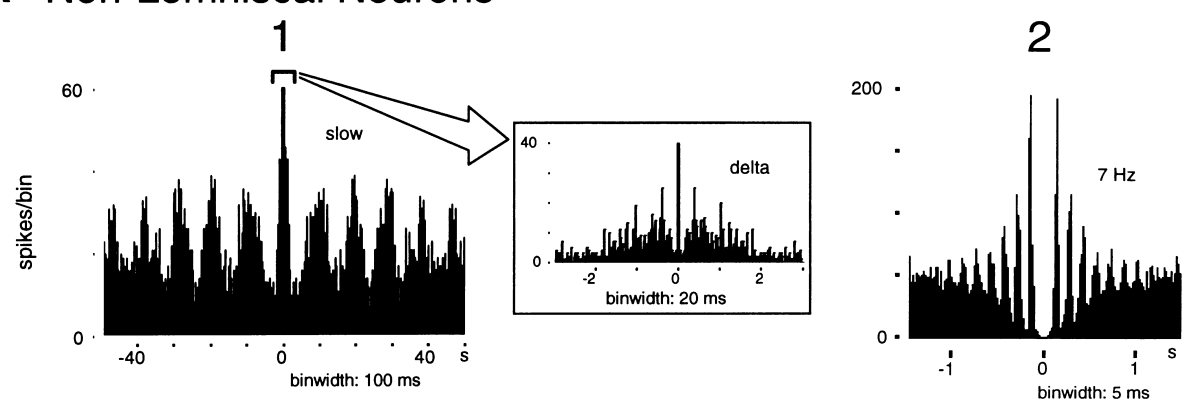

\section{B Lemniscal Neurons}
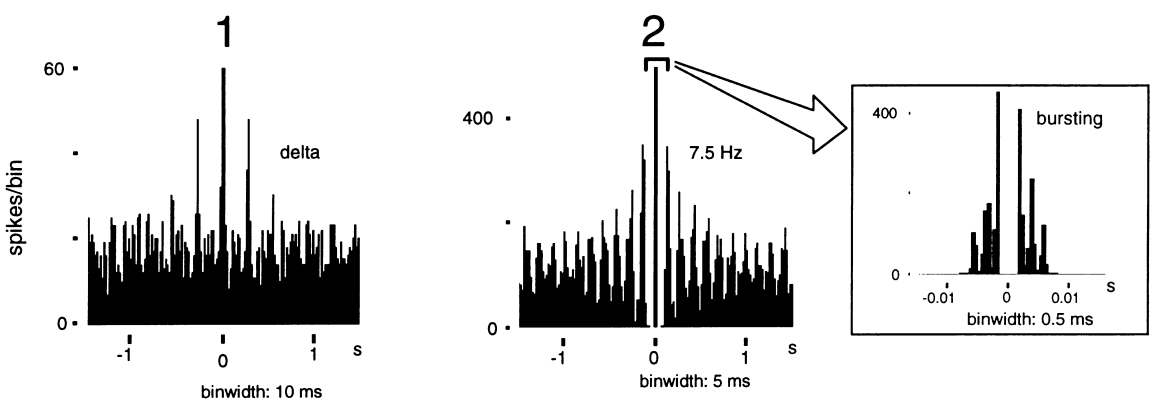

Fig. 3. Spontaneous rhythmicity. (A, B) Auto-correlograms computed from the single spikes of two cuneate non-projection neurons (A1, A2) and two projection cells $(\mathrm{B} 1, \mathrm{~B} 2)$.

spike-and-wave complexes ended the paroxysms. Repetitive tetani induced paroxysmal discharges when applied every 2 min or less frequently. Sample records obtained from the same animal in response to $100-\mathrm{Hz}$ iterative cortical stimulation applied successively from the medial precruciate cortex to the medial postcruciate cortex (electrode positions $1-6$ in Fig. 6A) at intervals of 5 min are depicted in Fig. 6B.

The cortical paroxysmal discharges were subcortically reflected. The paroxysms generated synchronized activity in the homolateral VPL and the contralateral cuneate, which was particularly evident at the end of the paroxysms, when they reached spike-and-wave discharges at $0.5-4 \mathrm{~Hz}$ (Fig. 7A). Additionally, mass recordings from both MLs (three animals) demonstrated that the cortical paroxysms were not transmitted bilaterally, but only to the lemniscus ipsilateral to the stimulated cortex (Fig. 7B).

\section{Lesion of the pyramidal tract}

To ascertain that the corticofugal pathway mediated the corticocuneate synchronization, the PT was electrolytically lesioned at mid-bulbar level (Fig. 8B). This prevented the appearance of the cortical synchronizing effect observed on both the contralateral cuneate and the ipsilateral ML. It was also consistently found that the background gross cuneate activity observed during the cortical paroxysm decreased in
PT-lesioned animals relative to non-lesioned animals, indicating that the cerebral cortex still had access to the cuneate, probably through the medial bulbopontine reticular formation. ${ }^{8,20,38,39}$ PT lesion also consistently abolished the appearance of the slow and spindle rhythmicities in the cuneate nucleus. The auto-correlograms of Fig. 8B illustrate that the slow rhythmic pattern observed in cuneate cells (autocorrelogram on the left) disappeared after lesioning the PT (auto-correlogram on the right). Finally, PT lesion also abolished the reflection of the cortical paroxysms on ipsilateral medial lemniscal single fibers (Fig. 8C), although some multiunitary background rhythmic activity coincident with the cortical spike-and-wave discharges was still present (Fig. 8C2), which was abolished by cortical removal (data not shown). The fast runs that initiated the paroxysm increased the lemniscal activity, while the poly-spikes and the spikeand-wave discharges that terminated the paroxysm ${ }^{46}$ sculptured the lemniscal activity by inducing silenced firing coincident with the cortical "waves" (Fig. 8C, left panel).

\section{The cuneate and lemniscal delta rhythmicity is preserved after removing the frontoparietal cortex}

Since the cuneate neurons show spontaneous rhythmic activity within the $\delta$ range (Fig. 3) and the corticocuneate synchronization, both spontaneous and paroxysmal (Fig. 12),

Table 1. Cells with rhythmic activity revealed by auto-correlation

\begin{tabular}{|c|c|c|c|c|c|c|c|}
\hline & \multirow[b]{2}{*}{ Total } & \multirow[b]{2}{*}{ With rhythmic activity } & \multicolumn{4}{|c|}{ Single rhythmicity } & \multirow{2}{*}{$\begin{array}{l}\text { Coupled rhythmicity } \\
\text { (slow }+ \text { delta })\end{array}$} \\
\hline & & & Slow & Delta & Spindle & $>15 \mathrm{~Hz}$ & \\
\hline CLs & 81 & $37(100 \%)$ & $3(8.1 \%)$ & $17(45.9 \%)$ & $8(21.6 \%)$ & $5(13.5 \%)$ & $4(10.8 \%)$ \\
\hline nCLs & 64 & $38(100 \%)$ & $6(15.8 \%)$ & $7(18.4 \%)$ & $7(18.4 \%)$ & $15(39.4 \%)$ & $3(7.9 \%)$ \\
\hline Total & 145 & $75(100 \%)$ & $9(12 \%)$ & $24(32 \%)$ & $15(20 \%)$ & $20(26.6 \%)$ & $7(9.3 \%)$ \\
\hline
\end{tabular}




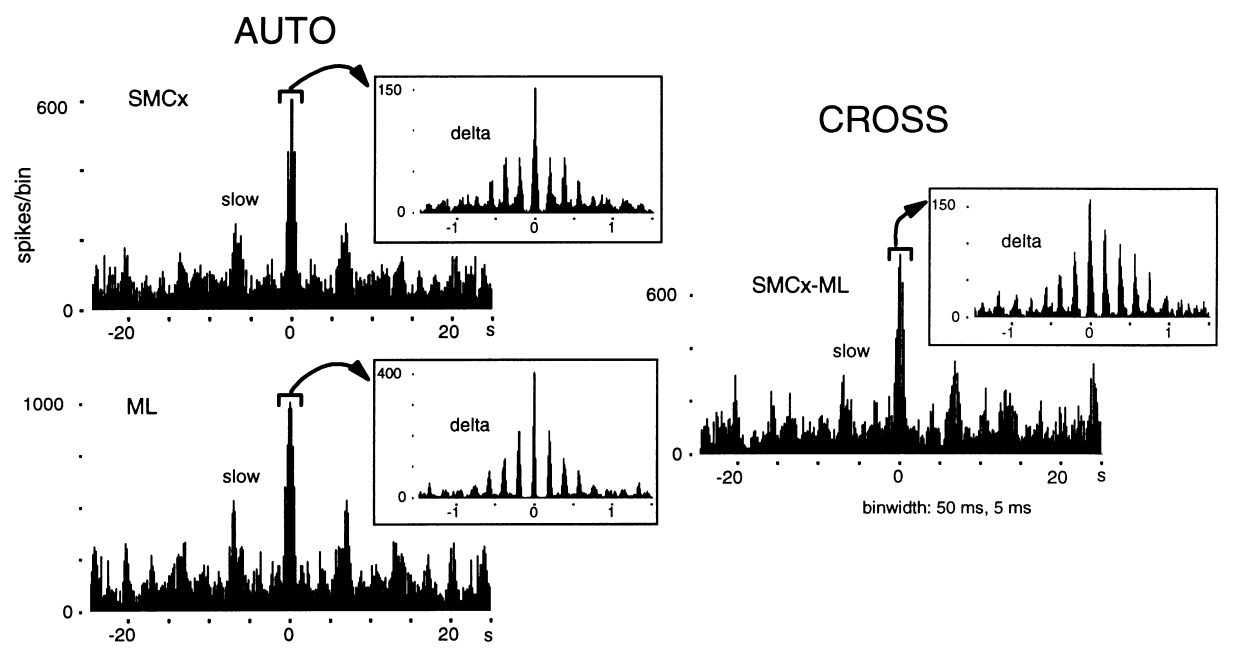

Fig. 4. Spontaneous synchronization. Auto-correlograms (left) of the unitary discharge of a sensorimotor cortical cell (SMCx) and a single lemniscal fiber (ML) recorded simultaneously, with the cross-correlogram shown on the right.

also occurs in the same band of frequencies, an obvious question was to ascertain whether this rhythmic pattern depends upon the integrity of the cerebral cortex. Accordingly, the frontoparietal cortex was removed in 11 animals (bilaterally in six of them) and simultaneous activity recorded from the cuneate and the ML (eight cats) or intracellularly from the cuneate (three cats). The results were consistent in all cats. A high proportion of CLs (21/35) and nCLs (16/29) showed rhythmic activity within the $\delta$ frequency. The remaining presented higher rhythmicities $(>15 \mathrm{~Hz})$, but none of them showed either slow $(<1 \mathrm{~Hz})$ or spindle oscillations. Examples of the rhythmicity presented by the cuneate neurons before and after bilateral removal of the frontoparietal cortex are illustrated by the intracellular records in
Fig. 9A and B, respectively. Membrane depolarization converted the oscillatory into tonic activity, as already described $^{7}$ (Fig. 9B).

The rhythmic activity observed in single medial lemniscal fibers paralleled the activity seen in the cuneate. Lemniscal fibers responding to cutaneous stimulation in the distal contralateral forelimb were selected, and thus were probably CLs. From a total of 25 well-discriminated single fibers, 14 had $\delta$ rhythmicity at rest. The example shown in Fig. 10 illustrates a multiunitary ML specimen record (left), from which the higher amplitude potential representing a single fiber was used to compile the auto-correlogram shown in the right panel. The fiber presented a spontaneous rhythmic activity within the $\delta$ range.

\section{SPINDLES}

$\mathrm{CN}$
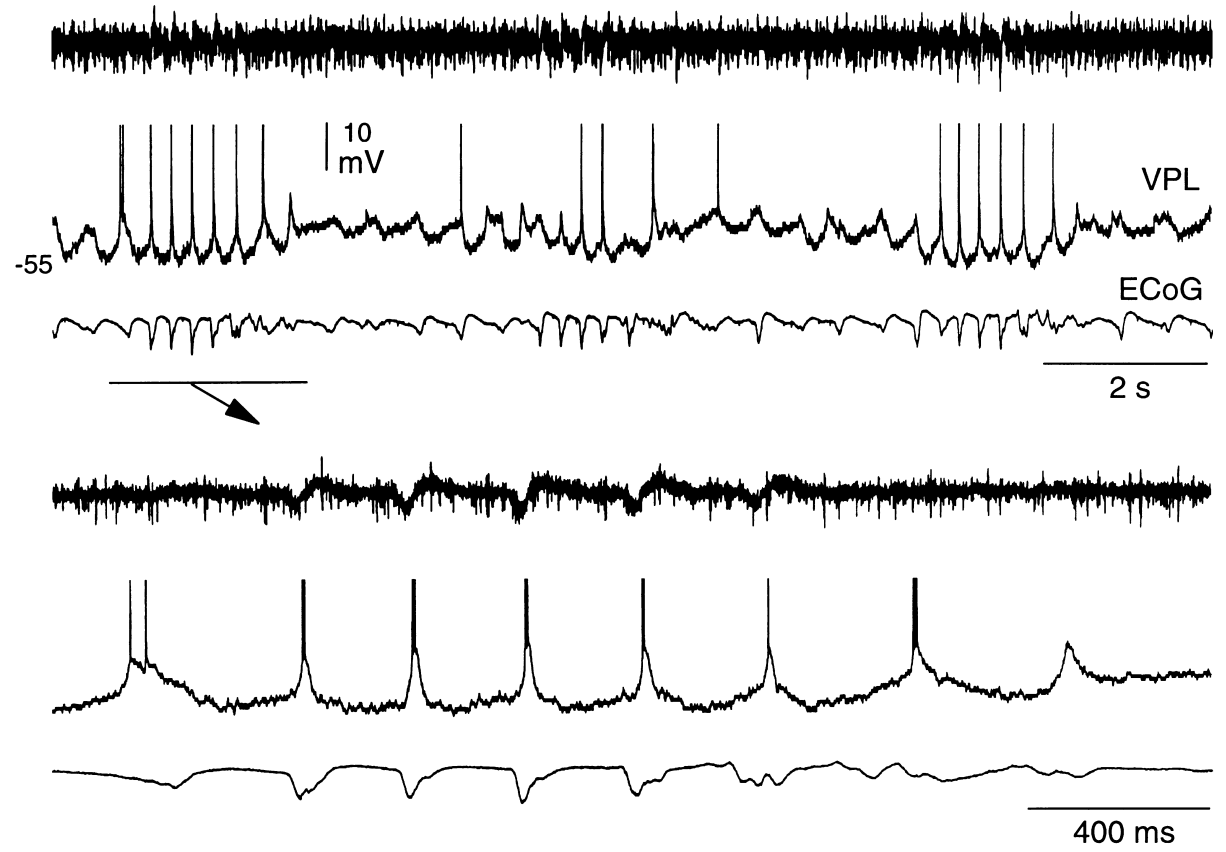

Fig. 5. The thalamocortical spindle oscillations are reflected in the cuneate nucleus. Simultaneous recordings from the VPL (intracellular) and from the middle cuneate nucleus $(\mathrm{CN}$; mass discharges) showed that the spindle rhythms were transmitted to the cuneate. The underlined sequence marked by a horizontal bar is expanded below. The VPL intracellular spikes are truncated. 


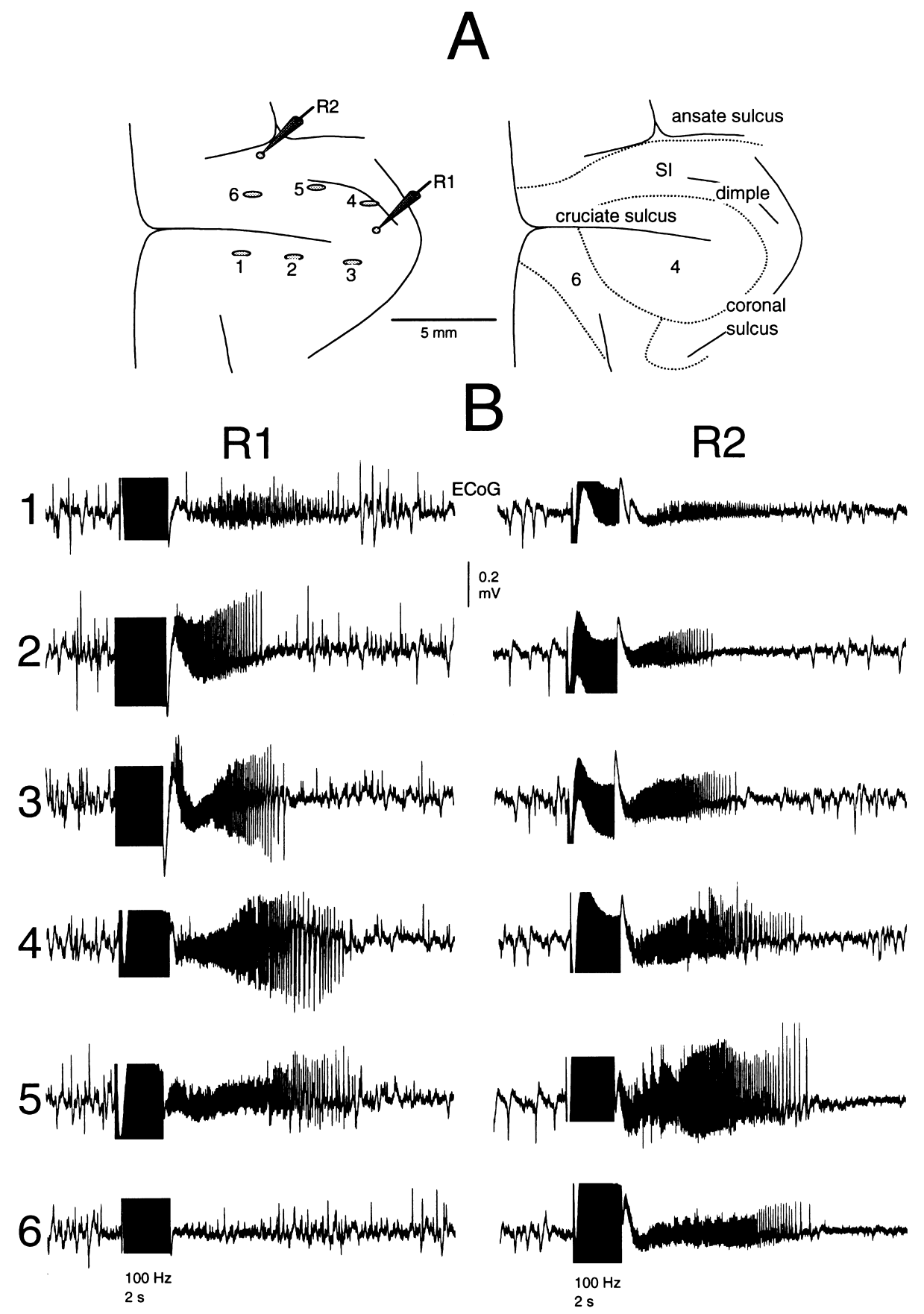

Fig. 6. Paroxysmal activity induced by electrical stimulation of the sensorimotor cortex. The diagrams (A) indicate the relative placement of six bipolar stimulating electrodes (1-6), as well as two electrocorticographic recording electrodes (R1, R2), with respect to the cortical sulci schematized on the right. (B) Sample records from electrodes R1 and R2 in response to bipolar stimulation through electrodes 1-6 obtained from the same animal.

\section{Post-inhibitory excitation}

Although the cerebral cortex imposes its rhythmic patterns on subcortical target structures, the cuneate neurons still showed oscillatory activity in the absence of cortical input. It is therefore possible that intrinsic membrane properties can partially explain this behavior. Since the presence of lowthreshold calcium potentials ${ }^{22,23}$ is usually associated with the capability to generate intrinsic rhythmicity in other central neurons ${ }^{50}$ we searched for post-anodal excitatory potentials in cuneate cells. Post-inhibitory rebound action potentials occurred spontaneously and could be experimentally induced in CLs and nCLs. Figure 11 shows sample records obtained from an nCL (Fig. 11A) and from two different CLs (Fig. $11 \mathrm{~B}, \mathrm{C})$. When the membrane potential of the nCL spontaneously hyperpolarized below $-65 \mathrm{mV}$, the neuron generated post-inhibitory rebound spikes (arrow in Fig. 11A). Transitory pulses of negative current induced post-inhibitory rebound potentials in two CLs (marked by arrows in Fig. $11 \mathrm{~B}$ and $\mathrm{C}$ ). These rebounds could be generated by increasing the duration and/or the intensity of the injected pulse (Fig. $11 \mathrm{C} 2, \mathrm{C} 3)$, thus demonstrating their time and voltage dependence. Depolarizing pulses led to the generation of full spikes, followed by neuronal accommodation (Fig. 11C). \{Table 2$\}$ 


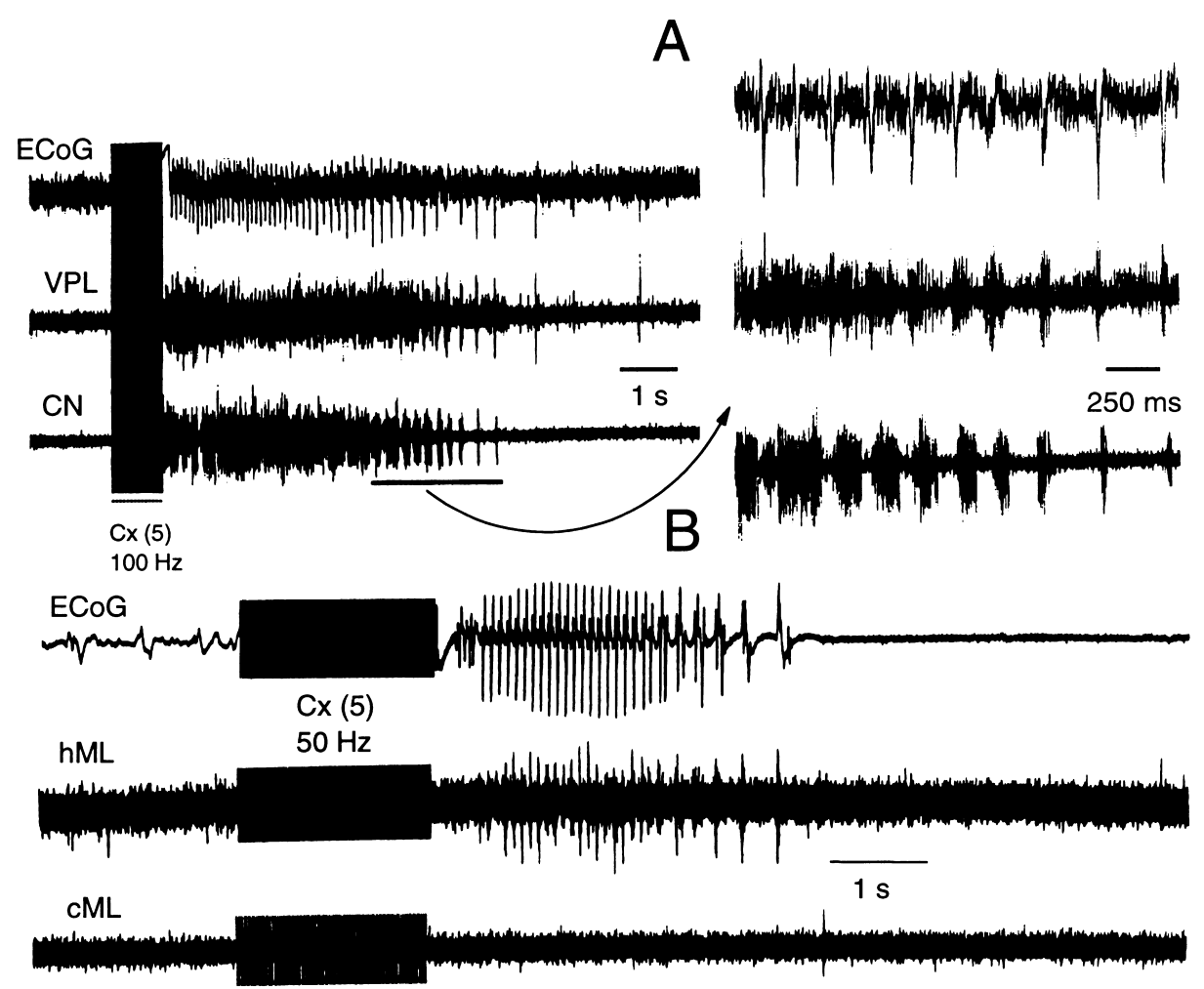

Fig. 7. The cortical paroxysms induced coupled activity in the contralateral cuneate nucleus (CN) and the ipsilateral VPL. (A) Fast cortical electrical stimulation generated coupled oscillatory activity in the cuneate nucleus and VPL; the underlined sequence is expanded on the right. (B) Bilateral multiunitary recordings from both MLs demonstrated that the paroxysmal activity was uniquely reflected on the lemniscus ipsilateral to the stimulated cortex.

Synaptic effects induced by the cortical paroxysm on cuneate neurons

It appears clear that there is a strong correlation between the cortex and the cuneate during sleep oscillations. However, intracellular recordings are necessary to evaluate the synaptic effects induced by the cortex on cuneate neurons. To maximize the probability of corticocuneate interactions, paroxysmal activity was electrically induced in the sensorimotor cortex while intracellularly recording from cuneate cells. From a total of 65 cuneate neurons tested, the great majority was affected by the cortical synchronization (Table 2). Surprisingly, during the stimulating periods and the early phase of the paroxysmal episodes, both classes of cuneate cells generated a sustained depolarization with increased tonic activity (Fig. 12A, C). Only towards the end of the paroxysms, when the electrocorticographic and the cuneate activities were highly synchronized, were the CLs and nCLs differentially affected. The records of Fig. 12 exemplify the behavior of the nCLs. Forelimb motor cortex stimulation $(100 \mathrm{~Hz} / 2 \mathrm{~s})$ induced EPSPs eventually crowned by spikes and a sustained depolarization with increased single activity that outlasted the end of the cortical tetanus by more than $5 \mathrm{~s}$ [Fig. 12A, the two lower panels are the expansions of the underlined sequences at the beginning of the cortical train (left panel) and at the end of the paroxysm (right panel)]. Only at the end of the sustained depolarizing shift (a portion expanded in Fig. 12C) did the ECoG and unit activity begin to be highly synchronized (Fig. 12D), and towards the end of the paroxysmal episode the cell discharged sequences of bursts at $\sim 3 \mathrm{~Hz}$ which were tightly coupled to the "spike" component of the cortical spike-and-wave complex (Fig. 12E).
Except that the CLs showed inhibitory postsynaptic potential (IPSPs), EPSPs or sequences of EPSPs-IPSPs during the cortical tetani, the effects observed during the early phase of the paroxysmal episodes were similar. The samples shown in Fig. 13 summarize the behavior of the nCLs (Fig. 13A) and CLs (Fig. 13B) during the late phase of the cortical paroxysm. While the totality of the presumed interneurons generated bursts of spikes coincident in time with the ECoG "spike" (Fig. 13A), most of the CLs (20/28) showed IPSPs with arrest of firing synchronized with the cortical "spike" (Fig. 13B1). The remaining CLs generated sequences of excitation-inhibition ( $n=5$; Fig. 13B2) or bursting activity $(n=3$; Fig. 13B3), also coincident with the "spike" of the ECoG. The time lags between the onset of the cortical "spike" and the synaptic potentials observed in the cuneate ranged from 5 to $22 \mathrm{~ms}(11.2 \pm 7.5 \mathrm{~ms}$, mean \pm S.D. $)$ for the EPSPs of the nCLs and from 10 to $17 \mathrm{~ms}(13.5 \pm 3.1 \mathrm{~ms})$ for the IPSPs of the CLs. Note that the CLs showing bursting activity timelocked to the electrocorticographic "spike" (Fig. 13B3) showed the smallest lag with respect to the onset of the cortical "spike", a fact that is congruent with the results obtained when applying low-frequency stimulation to the sensorimotor cortex (Canedo A. and Mariño J., unpublished observations).

\section{DISCUSSION}

\section{General}

Previous results demonstrated the existence of spontaneous oscillatory activity in the cuneate nucleus of chloralose-anesthetized cats, ${ }^{7,28}$ in the gracile nucleus of 
CONTROL

A

AFTER PT LESION
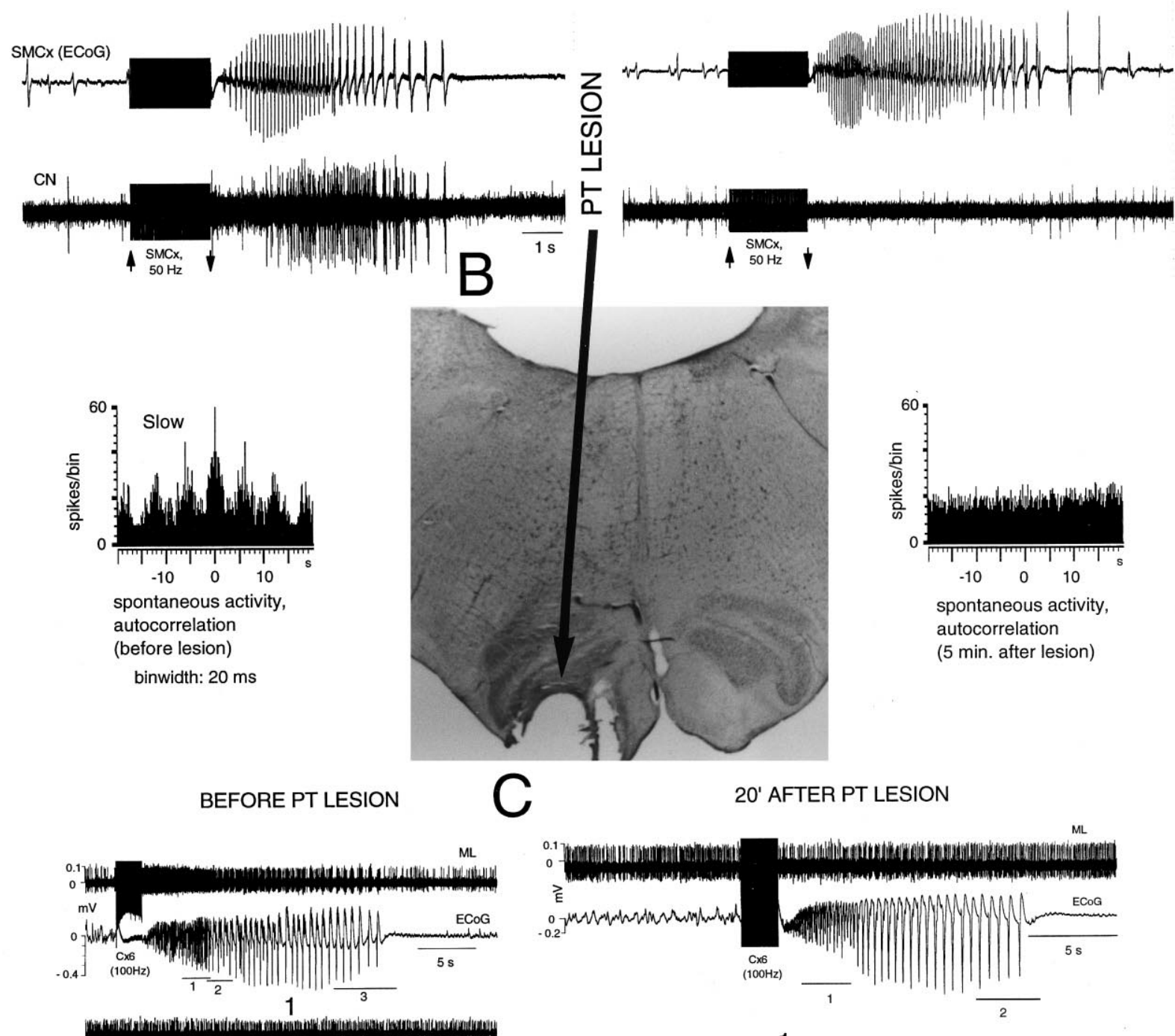

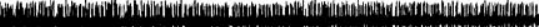
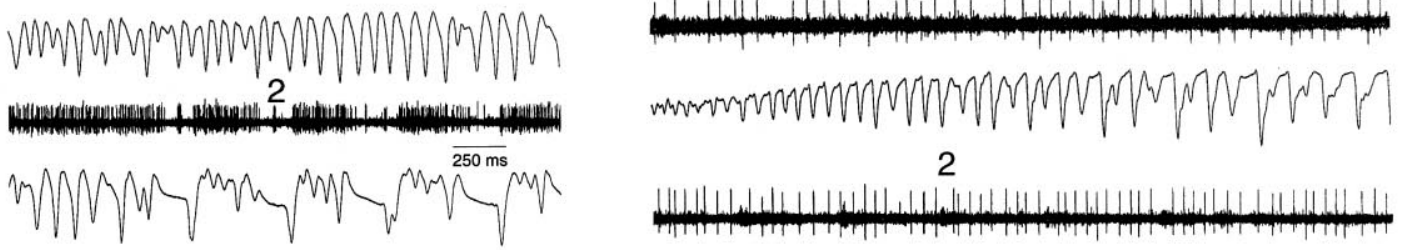

1

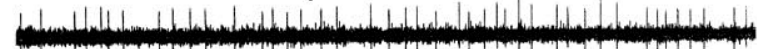

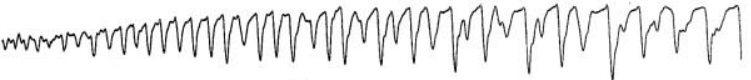

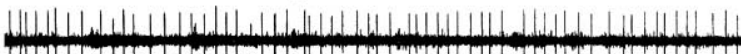

3
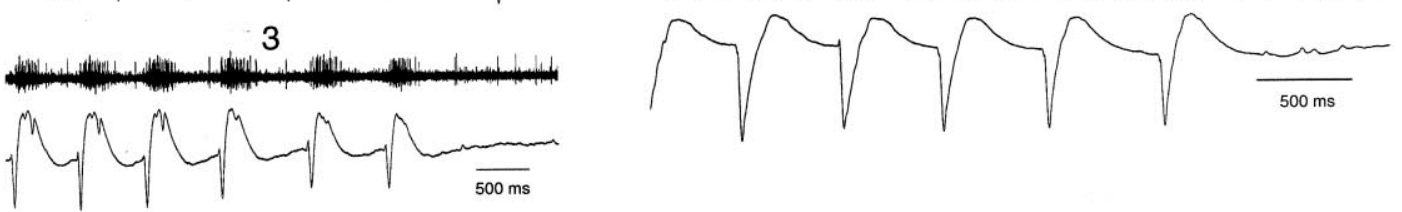

Fig. 8. Spread of cortical electrocorticographic activity to the cuneate nucleus and to the ML. The cortical synchronized activity imposed its rhythmic patterns over the contralateral cuneate nucleus (A, Control). The cortical synchronization imposed on the cuneate depended on the integrity of the PT since, after lesioning the tract (B; the arrow points to the lesion in the histological section), the cortical discharges were not reflected in the nucleus (A, lower record on the right). PT lesion also abolished the cuneate slow oscillations: the auto-correlograms shown to the left and right of the histological section (B) were obtained before and $5 \mathrm{~min}$ after the PT lesion, respectively, while maintaining the cuneate electrode in place. Detected events were the unitary extracellular spikes of the same cuneate neuron. (C) The reflection of the cortical paroxysm on the ipsilateral ML also depended on the integrity of the PT (C; the underlined numbered sequences are expanded below, as indicated).

pentobarbital- and urethane-anesthetized rats, ${ }^{32}$ and in DCN slices from young rats. ${ }^{31}$ This study further shows that, excepting the slow and spindle oscillations, the remaining rhythms survived lesion of the PT or bilateral removal of the frontoparietal cortex. In addition, it is demonstrated that the electrically elicited sensorimotor cortical paroxysms 


\section{A- Cortex Intact}

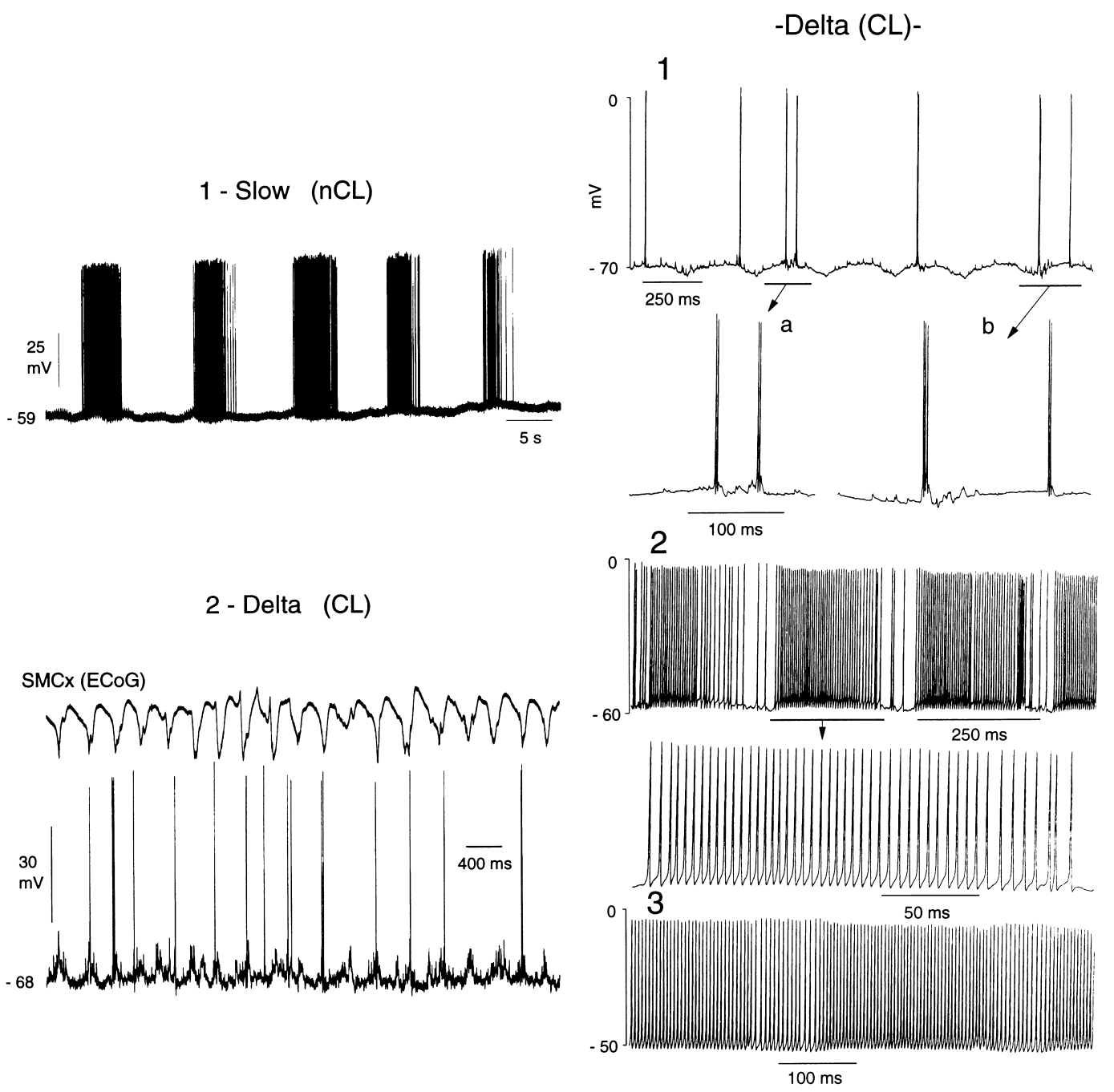

Fig. 9. The cuneate $\delta$ oscillations survived bilateral removal of the frontoparietal cortex. Two different cuneate neurons showing spontaneous rhythmic activity within the slow (A1) and $\delta$ (A2; note that the electrocorticographic and neuronal activity were tightly coupled) band. A different cuneate neuron (B) showed $\delta$ rhythmicity at rest (B1) after bilateral removal of the frontoparietal cortex. The portion expanded in 1b shows a triplet of spikes emerging from a slow hyperpolarization-activated potential. Gradual membrane depolarization induced single-spike, tonic activity (B2, B3). The portions marked by horizontal bars in $\mathrm{B} 1$ and $\mathrm{B} 2$ are expanded below, as indicated.

spread down to the cuneate, imposing coupled neuronal activity between the cortex itself and the cuneate neurons. The synchronization between cortical and cuneate activity progressively increased towards the end of the paroxysmal episodes, and at $0.5-4 \mathrm{~Hz}$, the cuneate neurons were synchronized with the ECoG. During the cortical "spike", most nCLs generated bursting discharges, while the majority of CLs were inhibited. The opposite occurred during the cortical "wave".

It may be argued that the cortical paroxysms are due to the effects of the chloralose, but fast electrical stimulation applied to the cerebral cortex or the dorsal thalamus of cats anesthetized with barbiturates or ketamine-xylazine induces paroxysmal activity with similar duration, as well as frequency and amplitude variations, as the ones reported in this study. ${ }^{54}$
Identification of cuneolemniscal and non-cuneolemniscal neurons

Although there is always the uncertainty that ML stimulation could fail to activate all CLs, differential intrinsic properties between CLs and nCLs have been reported with reasonable confidence when separating these two classes of cells. $^{2,7,31}$ It is, however, possible that some cuneate neurons project to other different sites of the ML, such as the cerebellum or the spinal cord, but the great majority of these cells are located outside the clustered region sampled in this study. ${ }^{3,5,9,16,25,57}$ Additionally, the cuneate neurons sending an axon to the contralateral pretectum and tectum via the ML are also located outside the clustered region. ${ }^{3,5}$ Thus, the CLs probably represent cuneothalamic neurons and the nCLs intrinsic local circuit, inhibitory cells (interneurons). 


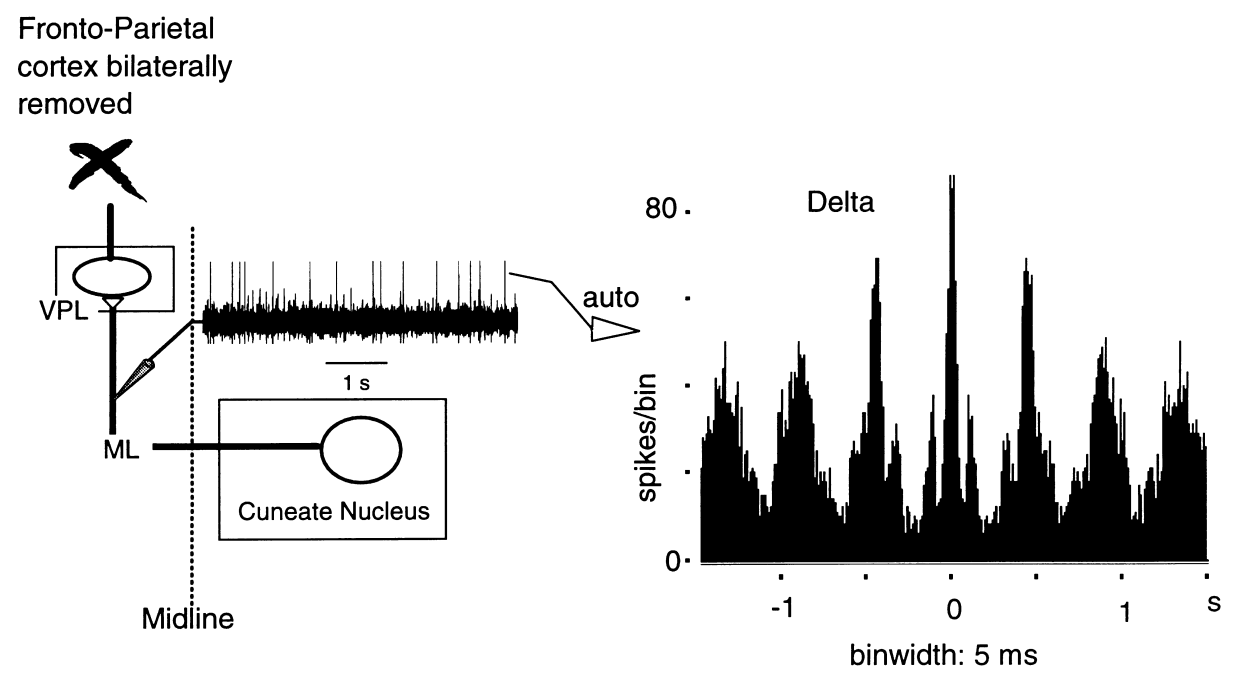

Fig. 10. The $\delta$ lemniscal rhythmicity also survived cortical removal. The recording electrode in the ML picked up a single well-isolated fiber from the background presenting rhythmic activity within the $\delta$ frequency after bilateral removal of the frontoparietal cortex (auto-correlogram on the right).

The presumed corticocuneate fibers responded with highfrequency spike bursts to cortical volleys (Fig. 2B). The cortical stimuli must have activated fast-rhythmic bursting cortical neurons that discharge high-frequency spike bursts at fast rates, ${ }^{41}$ and thus exert powerful synaptic influences on cuneate neurons. In fact, cortical stimulation induced bursting activity and summed EPSPs on cuneate neurons (Fig. 2A, lower trace). That the corticocuneate fibers discharge in high-frequency bursts correlated with cortical epileptiform discharges has also been reported in cats anesthetized with nitrous oxide (see fig. 4 in Ref. 37).

\section{Spontaneous rhythmicity}

The study of spontaneous oscillations in the anesthetized animal is of undoubted help in uncovering intrinsic neuronal properties and synaptic interactions. The majority of CLs have a resting or spontaneous discharge, ${ }^{31}$ respond with a
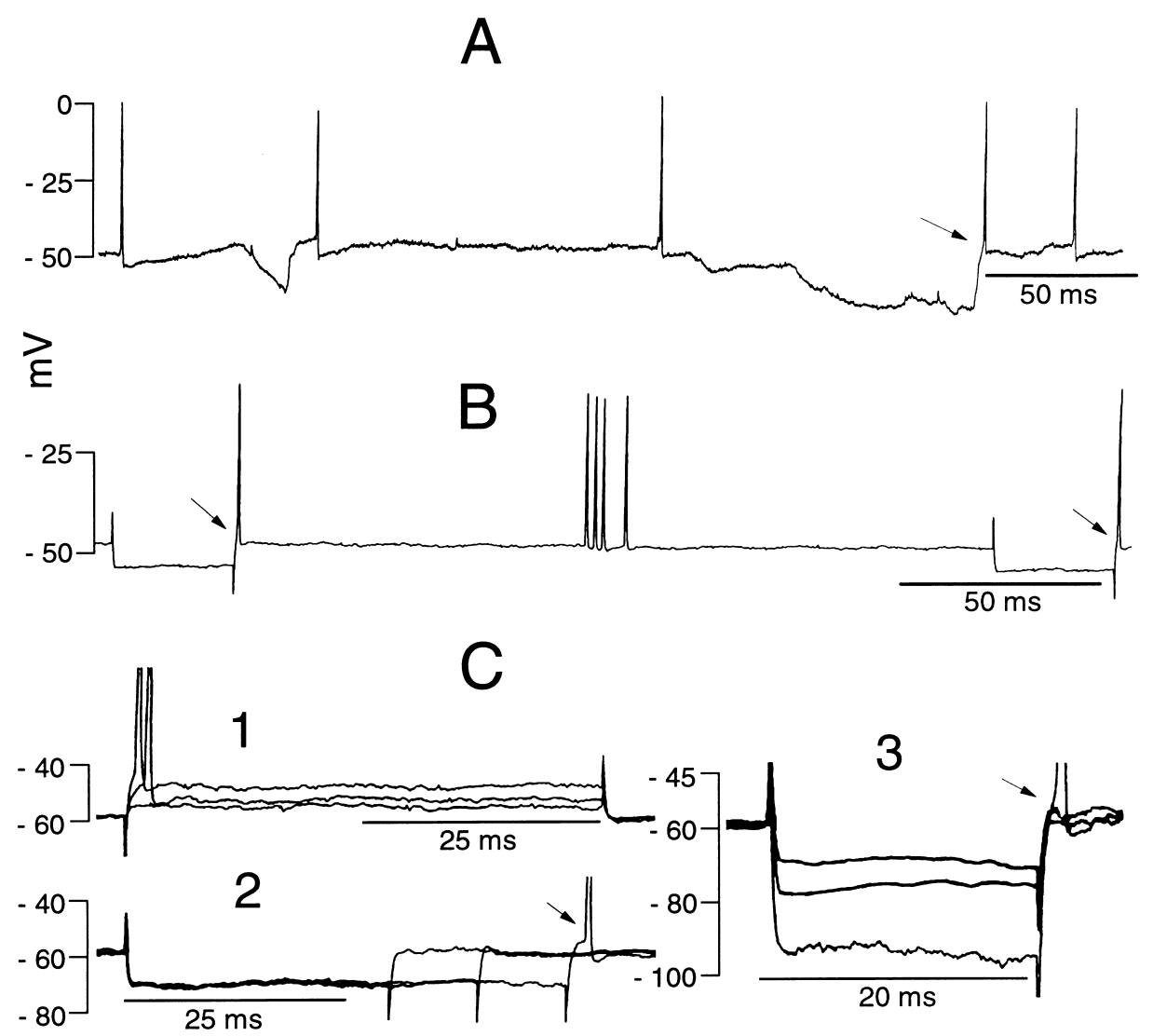

Fig. 11. Afterhyperpolarization rebound spikes. (A) An nCL showing spontaneous hyperpolarizations, one of which induced a rebound spike (signaled by an arrow). (B, C) Two different CLs showing post-anodal excitation (marked by arrows) which were time (C2) and voltage (C3) dependent. Depolarizing pulses induced full spikes at the beginning, with ulterior accommodation (C1). 
Table 2. Effects induced by the cortical paroxysm on cuneate cells

\begin{tabular}{lccccc}
\hline & Tested & IPSPs & EPSP-IPSPs & Bursting & Not affected \\
\hline $\mathrm{Cl}$ & 36 & $20(55.5 \%)$ & $5(13.9 \%)$ & $3(8.3 \%)$ & $8(22.2 \%)$ \\
$\mathrm{nCL}$ & 29 & - & - & $29(100 \%)$ & $-\overline{-}$ \\
Total & 65 & $20(30.7 \%)$ & $5(7.7 \%)$ & $32(49.2 \%)$ & $8(12.3 \%)$ \\
\hline
\end{tabular}

high-frequency burst of spikes to a single electrical stimulus and can generate bursting activity in the absence of afferent input. ${ }^{4,17,31,34}$ The resting discharge and the propensity to fire in bursts may be explained by sustained afferent input, through intrinsic neuronal properties and by continuous recurrent and cortical bombardment (Canedo A. and Mariño J., unpublished observations).

The cuneate slow and spindle oscillations are imposed by the cerebral cortex through the PT, since lesion of this tract (Fig. 8B) or removal of the frontoparietal cortex abolished them. The cortically generated slow oscillation is transmitted to the thalamus, to the striatum, to the pedunculopontine nucleus, to the amygdala (see references in Ref. 49) and to the cuneate (this work). It is present in unanesthetized cats, ${ }^{1,52}$ and in ketamine-xylazine, urethane ${ }^{51}$ and chloralose (present work) anesthetized animals. The slow oscillation is synchronously observed in reticular thalamic and thalamocortical cells, ${ }^{47}$ and in many distant and functionally different neocortical areas, including the motor and visual cortices. ${ }^{1}$ According to the present data, the neocortex oscillates as a whole at slow $(<1 \mathrm{~Hz})$ frequencies, as do subcortical neuronal ensembles targeted by direct cortical input.

Since the spindle activity recorded in the cuneate did not survive PT lesion or cortical removal, the cuneate inhibitory interneurons surrounding the CLs do not have the capability to intrinsically generate spindle activity. Rather, the spindles generated in the reticularis thalami nucleus are translated to the cerebral cortex by the thalamocortical cells. The cortical
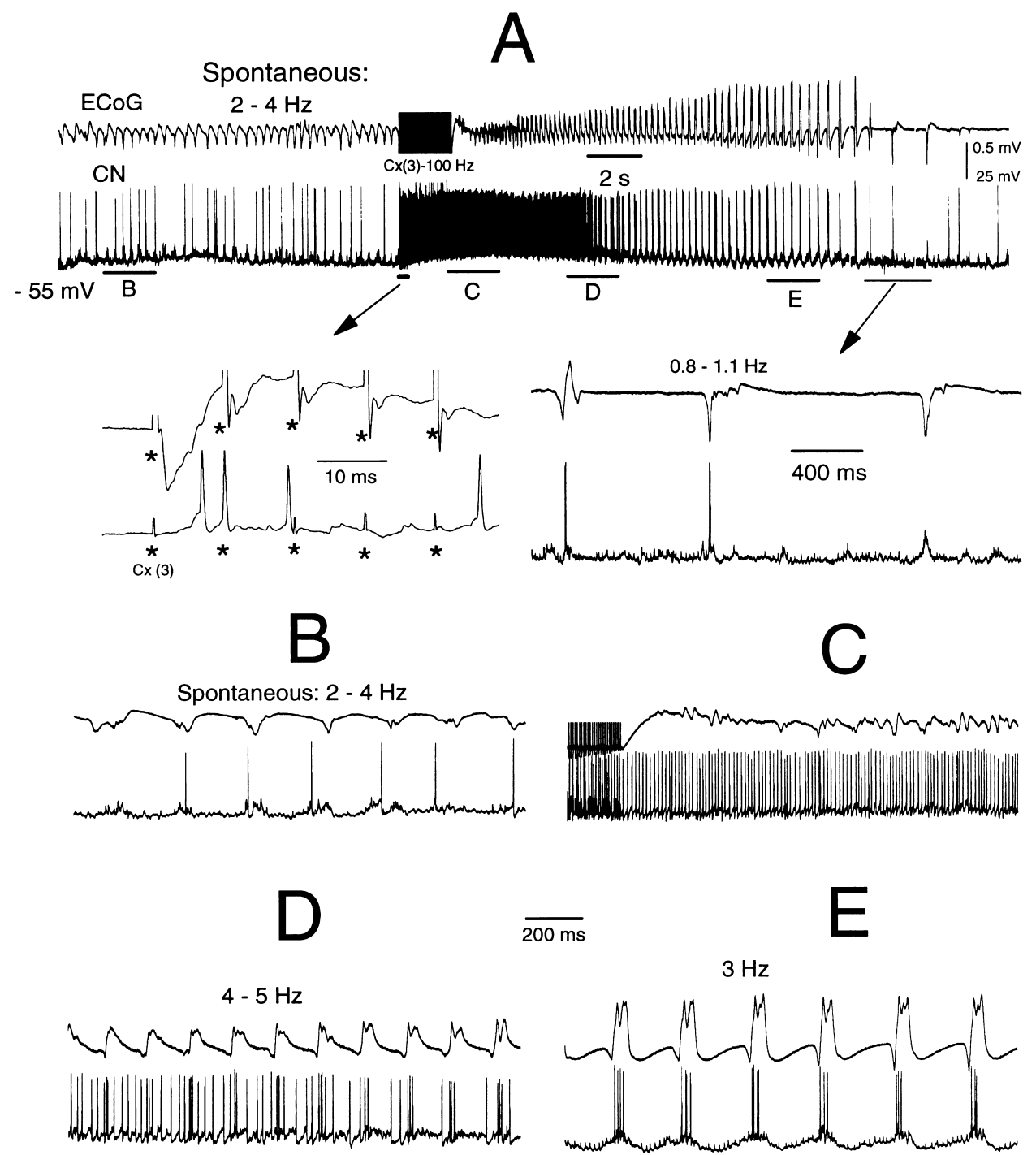

Fig. 12. The cortical paroxysm gradually imposed its late rhythmic patterns on the cuneate. (A) Simultaneous recordings of the ECoG and of a cuneate nonlemniscal neuron $(\mathrm{CN})$. Under deep chloralose anesthesia, the ECoG showed spontaneous negative "spikes" within the $\delta$ frequency tightly coupled to the cuneate neuronal activity (see the underlined sequence expanded in B). High-frequency (100 Hz/2 s) motor cortex stimulation [Cx(3)] induced EPSPs eventually crowned by spikes (see the underlined sequence at the onset of the train), and steadily depolarized the cell, leading to a tonic firing increase lasting about $5 \mathrm{~s}$ (see underlined sequence expanded in C). After membrane repolarization and towards the end of the paroxysm, the coupling between the ECoG and the cuneate neuronal activity progressively increased (see the underlined sequences expanded in D and E) until the cerebral cortex totally imposed its $\delta$ pattern of activity $(\mathrm{E})$. 
A

NON-CUNEOLEMNISCAL NEURON

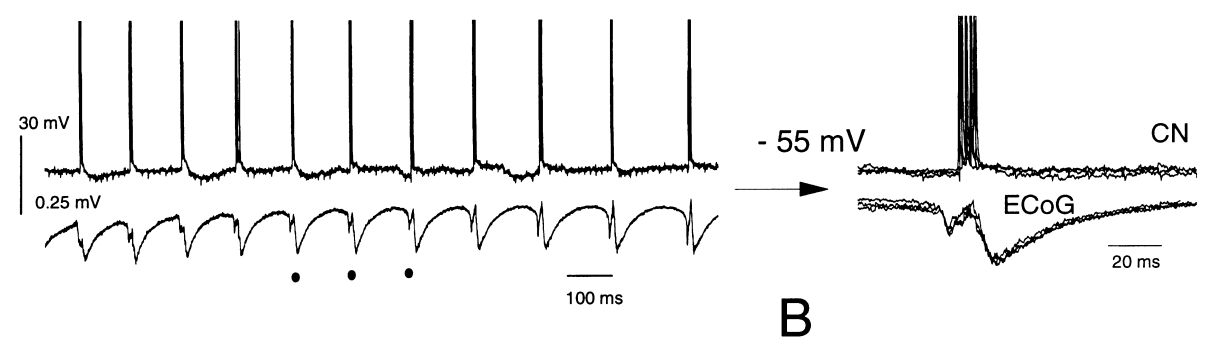

1

CUNEOLEMNISCAL NEURONS
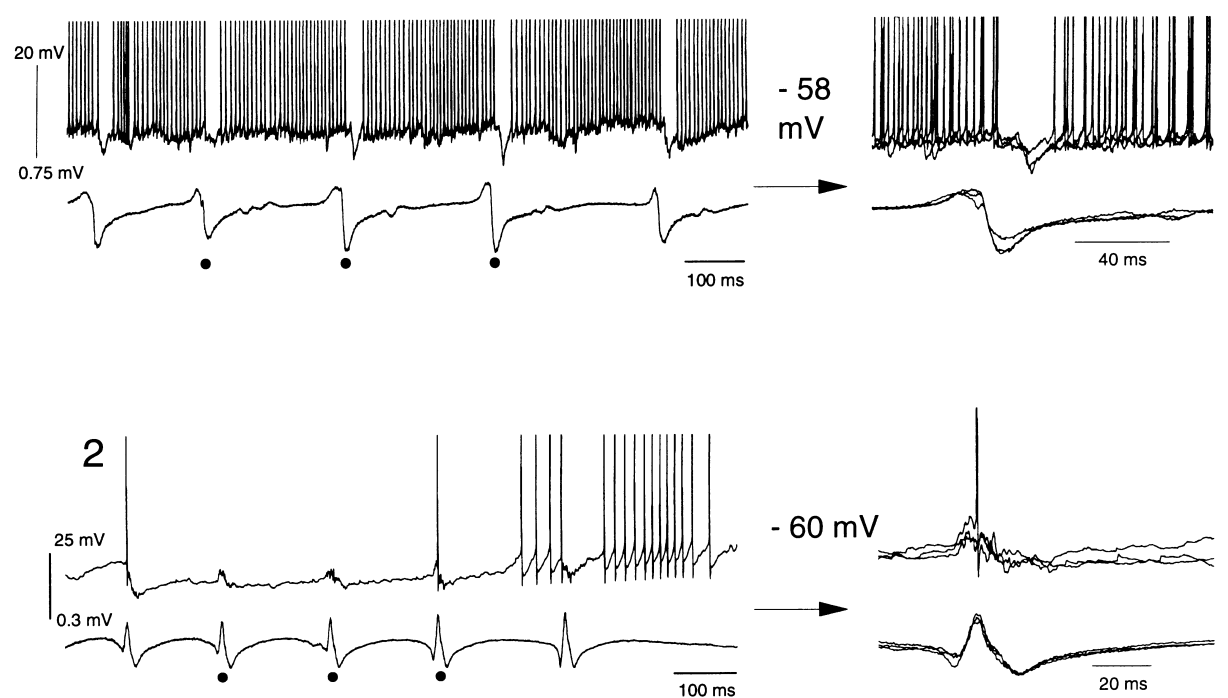

3

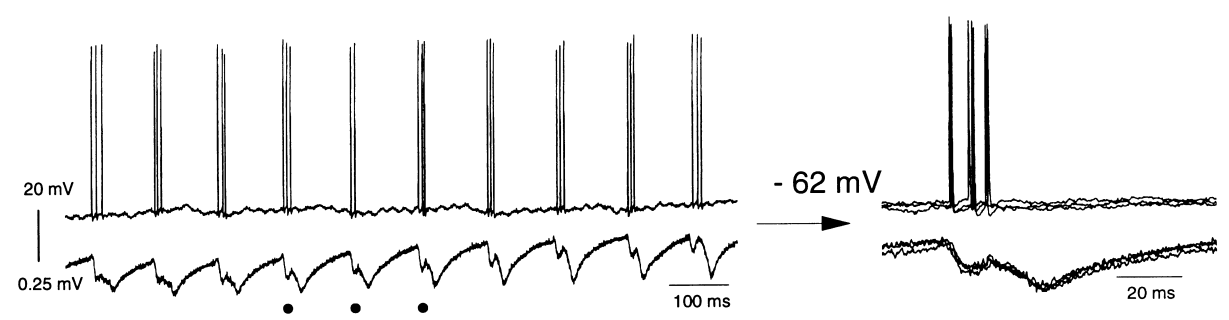

Fig. 13. General effects exerted by the cortical paroxysms on cuneate neurons. The records shown exemplify the observed synchronization between the ECoG and cuneate cells at the late phase of the paroxysmal episodes for both non-lemniscal (A) and lemniscal neurons (B1-B3). The events marked by black dots are superimposed on the right for each pair of traces. The spikes in B1 and B2 are truncated.

PT cells, in turn, spread down this rhythmic pattern to the cuneate. One of the functions of the spindle oscillations appears to be the disconnection from the external environment, further enhanced by the $\delta$ oscillations during quiet sleep. ${ }^{41}$ Such a disconnection from the somatosensory external world may be produced at the very early processing of incoming information, within the DCN, probably as an effect of spindles transmitted to the cuneate by the PT and enhanced through intrinsic mechanisms. It is well known that, during quiet sleep and deep anesthesia, the thalamo-cortico-thalamic networks show oscillatory bursting activity. The cuneate neurons coherently oscillate within the same band of frequencies, thus avoiding disrupting or converting the oscillatory activity of the thalamo-cortico-thalamic network to the tonic mode. The neocortex imposes its patterns of activity on subcortical neurons so that the thalamo-cortico-thalamic and prethalamic cells resonate. Consequently, during the states of sleep and anesthesia, the cortex and the structures it directly targets may generate a global functional internal state isolating the organism from its external environment, thus avoiding the processing of sensory inputs. On the contrary, during wakefulness, the tonic mode of activity of the cortico-thalamo-cortical network will exert a smaller and more concentrated influence on the DCN, allowing faithful relay of specific sensory inputs by the modulation exerted by corticofugal neurons selectively activated by the somatosensory inputs reaching the cortex.

The $\delta$ and higher rhythmicities (excluding the spindle oscillations) survived the lesion of the PT or bilateral removal of the frontoparietal cortex (Figs 9B, 10). This indicates that, in 
addition to reflecting cortical oscillations, the cuneate retains the capacity to sustain oscillatory activity whether imposed from extracortical sources or generated intrinsically (Figs 911; also see Refs 7 and 31). In particular, both CLs and nCLs showed a high incidence of spontaneous $\delta$ rhythms in the absence of corticocuneate input (Fig. 9B). This probably indicates that the $\delta$ oscillation is a characteristic feature of cuneate neurons, at least under chloralose anesthesia. The intracuneate circuitry, together with the intrinsic membrane neuronal properties, may sustain the rhythmic patterns observed in the absence of the frontoparietal cortex. The inhibitory interneurons surrounding the CLs and intermingled between the clusters of the middle cuneate may have a resetting effect by inhibiting, synchronizing and inducing rhythmic activity in ensembles of CLs.

\section{Spread of cortical paroxysms}

The oscillatory paroxysmal activity represents a massive neuronal synchronization characteristic of a pathophysiological process. It is, however, useful to experimentally study the corticofugal influences exerted on anatomically targeted neurons. Although the repetition rate of paroxysms (2-5 min) is consistent with the time-course of recovery from cortical spreading depression, the paroxysms were not consistently followed by depressed electrocorticographic activity. Similar results have been reported from cats under barbiturate or ketamine-xylazine anesthesia. ${ }^{54}$

The cortical paroxysms built up gradually, entraining progressive synaptic synchrony within the cortex and/or cortico-thalamo-cortical networks, ${ }^{42,54}$ and were reflected on cuneate and VPL neurons, as well as on lemniscal fibers (Figs 7, 8, 12, 13). Some background rhythmic activity coupled to the cortical paroxysm was still present in the ML after PT lesion (Fig. 8C2, right panel), indicating that the cortex still had access to lemniscal-projecting neurons, probably through the bulbar reticular formation.

The cuneate paroxysmal discharges were time-locked to the "spike" component of cortical field potentials, while silenced firing was related to the longer-lasting "wave" component, in particular at the end of the paroxysm (Fig. 12). The slowing of the corticocuneate network spike-andwave paroxysm to a frequency that matches the preferred frequency of cuneate cells may explain the synchronization observed between the ECoG and the cuneate activity, as proposed recently for thalamic networks. ${ }^{36}$

The synchronizing process progressively increased, the cuneate discharges lagged the onset of the "spike" in the ECoG, and both cortical and cuneate paroxysmal activities ended abruptly and synchronously. These findings indicate that the cortex imposed its oscillatory paroxysmal activity on the cuneate, which does not possess the necessary local circuitry to generate paroxysms by itself. Otherwise, the cuneate synchronization will outlast the cortical paroxysm. The reinforcement of the cortical paroxysm by the cuneate is unlikely, at least in the anesthetized animal, since many of the thalamocortical cells remain tonically hyperpolarized during the cortical paroxysm. ${ }^{45}$ On the contrary, the coupled resting activities within the $\delta$ band between cuneothalamic and thalamocortical cells may play a role in the reinforcement of the thalamo-cortico-thalamic clock-like oscillations.

The spike-and-wave paroxysms at $2-4 \mathrm{~Hz}$ may develop from 7 - to $14-\mathrm{Hz}$ spindle waves that often occur without apparent discontinuity from sleep oscillations, ${ }^{53}$ with the cortex having a leading role in their development. ${ }^{49}$ The experimental paroxysms obtained by direct cortical stimulation also began within the same range of frequencies, thus constituting a suitable experimental model. The cortical tetani basically induced the same responses on both CLs and nCLs during the early phase of the paroxysms, although only about $38 \%$ of projection neurons appear to receive excitatory cortical input (Canedo A. and Mariño J., unpublished observations). This may indicate that $\mathrm{GABA}_{\mathrm{A}}$ receptors, known to be abundantly represented in the cuneate, ${ }^{24,33,55,56}$ can also induce depolarizing responses..$^{35,40}$

It has been repeatedly reported that, upon cortical stimulation, the extracellular firing of cuneate and gracile neurons shows an initial increase followed by a longer period of firing decrease, further followed by a "rebound" facilitation. ${ }^{11,18}$ Under our experimental conditions, the cortical excitatory effects on CLs were the fastest observed (Fig. 2A), and thus the rapid monosynaptic excitation of CLs, together with post-inhibitory rebound potentials, probably induced through interneurons, explain the synchronization observed between the "spike" of the ECoG and the gross lemniscal activity (Figs 7B, 8C, left panel). It has been demonstrated that the fast-rhythmic bursting cortical neurons contribute to the initiation of spontaneous and electrically elicited spike-and-wave cortical paroxysms, ${ }^{44}$ and since the axons of these cells reach the cuneate (Fig. 2B), they might be responsible for the initial powerful excitatory effects exerted by the cerebral cortex on CLs, as reported previously. ${ }^{19,20}$ Concurrent activation of inhibitory interneurons would subsequently inhibit the CLs, which could intrinsically generate further rebound excitations brought about by hyperpolarization-activated $^{7,31}$ and low-threshold calcium conductances.

Acknowledgements - The technical assistance of Mr Francisco García Pablo is greatly appreciated. This work was supported by a grant from the DGICYT (PB96-0958).

\section{REFERENCES}

1. Amzica F. and Steriade M. (1995) Short- and long-range neuronal synchronization of the slow (<1 Hz) cortical oscillation. J. Neurophysiol. 73, 20-38.

2. Andersen P., Eccles J. C., Schmidt R. F. and Yokota T. (1964) Identification of relay cells and interneurons in the cuneate nucleus. J. Neurophysiol. 27, 1080-1095.

3. Berkley K. J., Blomqvist A., Pelt A. and Flink R. (1980) Differences in the collateralization of neuronal projections from the dorsal column nuclei and lateral cervical nucleus to the thalamus and tectum in the cat: an anatomical study using two different double-labeling techniques. Brain Res. 202, 273-290.

4. Brown A. G., Gordon G. and Kay R. H. (1974) A study of single axons in the cat's medial lemniscus. J. Physiol., Lond. 236, $225-246$.

5. Bull M. S. and Berkley K. J. (1984) Differences in the neurons that project from the dorsal column nuclei to the diencephalon, pretectum, and tectum in the cat. Somatosensory Res. 1, 281-300.

6. Canedo A. and Mariño J. (1998) Corticofugal effects on somatosensory ascendant transmission in the cuneate nucleus of chloralose-anaesthetized cats. J. Physiol., Lond. 509P, 31S-32S.

7. Canedo A., Martinez L. and Mariño J. (1998) Tonic and bursting activity in the cuneate nucleus of the chloralose anesthetized cat. Neuroscience 84, 603-617. 
8. Cesa-Bianchi M. G. and Sotgiu M. L. (1969) Control by brain stem reticular formation of sensory transmission in Burdach nucleus. Analysis of single units. Brain Res. 13, 129-139.

9. Cheek M. D., Rustioni A. and Trevino D. L. (1975) Dorsal column nuclei projections to the cerebellar cortex in cats as revealed by the use of the retrograde transport of horseradish peroxidase. J. comp. Neurol. 164, 31-46.

10. Cheema S., Rustioni A. and Whitsel B. L. (1985) Sensorimotor cortical projections to the primate cuneate nucleus. J. comp. Neurol. 240, 196-211.

11. Cole J. D. and Gordon G. (1983) Timing of corticofugal actions on the gracile and cuneate nuclei of the cat. J. Physiol., Lond. 340, $139-152$.

12. Contreras D., Destexhe A., Sejnowski T. J. and Steriade M. (1996) Control of spatiotemporal coherence of a thalamic oscillation by corticothalamic feedback. Science 274, 771-774.

13. Contreras D., Destexhe A., Sejnowski T. J. and Steriade M. (1997) Spatio-temporal patterns of spindle oscillations in cortex and thalamus. J. Neurosci. 17, 1179-1196.

14. Contreras D. and Steriade M. (1997) Synchronization of low-frequency rhythms in corticothalamic networks. Neuroscience 76, 11-24.

15. Destexhe A., Contreras D. and Steriade M. (1998) Mechanisms underlying the synchronizing action of corticothalamic feedback through inhibition of thalamic relay cells. J. Neurophysiol. 79, 999-1016.

16. Enevoldson T. P. and Gordon G. (1984) Spinally projecting neurons in the dorsal column nuclei: distribution, dendritic trees and axonal projections. A retrograde HRP study in the cat. Expl Brain Res. 54, 538-550.

17. Galindo A., Krnjevic K. and Schwartz S. (1968) Patterns of firing in cuneate neurons and some effects of Flaxedil. Expl Brain Res. 5, 87-101.

18. Gordon G. and Jukes M. G. M. (1964) Dual organization of the exteroceptive components of the cat's gracile nucleus. J. Physiol., Lond. 173, 263-290.

19. Gordon G. and Jukes M. G. M. (1964) Descending influences on the exteroceptive organizations of the cat's gracile nucleus. J. Physiol., Lond. 173, 291-319.

20. Jabbur S. J. and Towe A. L. (1961) Cortical excitation of neurons in dorsal column nuclei of cat, including an analysis of pathways. J. Neurophysiol. 24, 499-509.

21. Llinás R. and Ribary U. (1993) Coherent 40-Hz oscillation characterizes dream state in humans. Proc. natn. Acad. Sci. U.S.A. 90, 2978-2981.

22. Llinás R. and Yarom Y. (1981) Electrophysiology of mammalian inferior olivary neurons in vitro. J. Physiol., Lond. 315, 549-567.

23. Llinás R. and Yarom Y. (1981) Properties and distribution of ionic conductances generating electroresponsiveness of mammalian inferior olivary neurons in vitro. J. Physiol., Lond. 315, 569-584.

24. Lue J. H., Shieh J. Y., Wen C. Y., Chen K. N. and Chan S. A. (1994) GABAergic boutons establish synaptic contacts with the soma and dendrites of cuneothalamic relay neurons in the rat cuneate nucleus. Expl Brain Res. 98, 13-20.

25. Mantle-St John L. A. and Tracey D. J. (1987) Somatosensory nuclei in the brainstem of the rat: independent projections to the thalamus and cerebellum. J. comp. Neurol. 255, 259-271.

26. Mariño J. and Canedo A. (1997) Corticofugal neurons induce oscillatory responses in cuneo-thalamic cells. Soc. Neurosci. Abstr. $23,2079$.

27. Mariño J. and Canedo A. (1998) Intracellular evidence of cortico-cuneate synchronization during evoked spike-and-wave cortical seizure activity. J. Physiol., Lond. 509P, 180P.

28. Mariño J., Martinez L. and Canedo A. (1996) Coupled slow and delta oscillations between cuneothalamic and thalamocortical neurons in the chloralose anesthetized cat. Neurosci. Lett. 219, 107-110.

29. Martinez L., Lamas J. A. and Canedo A. (1995) Pyramidal tract and corticospinal neurones with branching axons to the dorsal column nuclei of the cat. Neuroscience 68, 195-206.

30. Núñez A., Amzica F. and Steriade M. (1992) Voltage-dependent fast (20-40 Hz) oscillations in long-axoned neocortical neurons. Neuroscience 51, $245-258$.

31. Núñez A. and Buño W. (1999) In vitro electrophysiological properties of rat dorsal column nuclei neurons. Eur. J. Neurosci. 11, $1865-1876$.

32. Panetsos F., Núñez A. and Avendaño C. (1997) Electrophysiological effects of temporary deafferentation on two characterized cell types in the nucleus gracilis of the rat. Eur. J. Neurosci. 9, 563-572.

33. Popratiloff A., Valtschanoff J. G., Rustioni A. and Weinberg R. J. (1996) Colocalization of GABA and glycine in the rat dorsal column nuclei. Brain Res. 706, 308-312.

34. Pubols B. H. Jr, Haring J. H. and Rowinski M. J. (1989) Patterns of resting discharge in neurons of the racoon main cuneate nucleus. J. Neurophysiol. 61, $1131-1141$.

35. Sánchez-Vives M. V., Bal T. and McCormick D. A. (1997) Inhibitory interactions between perigeniculate GABAergic neurons. J. Neurosci. 17, 8894-8908.

36. Sánchez-Vives M. V. and McCormick D. A. (1997) Functional properties of perigeniculate inhibition of dorsal lateral geniculate nucleus thalamocortical neurons in vitro. J. Neurosci. 17, 8880-8893.

37. Schwartzkroin P. A., Van Duijn H. and Prince D. A. (1974) Effects of projected cortical epileptiform discharges on unit activity in the cat cuneate nucleus. Expl Neurol. 43, 106-123.

38. Sotgiu M. L. and Margnelli M. (1976) Electrophysiological identification of pontomedullary reticular neurons directly projecting into dorsal column nuclei. Brain Res. 103, 443-453.

39. Sotgiu M. L. and Marini G. (1977) Reticulo-cuneate projections as revealed by horseradish peroxidase axonal transport. Brain Res. 128, 341-345.

40. Staley K. J., Soldo B. L. and Proctor W. R. (1995) Ionic mechanisms of neuronal excitation by inhibitory GABAa receptors. Science 269, 977-981.

41. Steriade M. (1997) Synchronized activities of coupled oscillators in the cerebral cortex and thalamus at different levels of vigilance. Cerebral Cortex 7, 583-604.

42. Steriade M. and Amzica F. (1994) Dynamic coupling among neocortical neurons during evoked and spontaneous spike-wave seizure activity. J. Neurophysiol. 72, 2051-2069.

43. Steriade M., Amzica F. and Contreras D. (1996) Synchronization of fast (30-40 Hz) spontaneous cortical rhythms during brain activation. J. Neurosci. 16, 392-417.

44. Steriade M., Amzica F., Neckelmann D. and Timofeev I. (1998) Spike-wave complexes and fast components of cortically generated seizures. II. Extraand intracellular patterns. J. Neurophysiol. 80, 1456-1479.

45. Steriade M. and Contreras D. (1995) Relations between cortical and thalamic cellular events during transition from sleep patterns to paroxysmal activity. J. Neurosci. 15, 623-642.

46. Steriade M. and Contreras D. (1998) Spike-wave complexes and fast components of cortically generated seizures. I. Role of neocortex and thalamus. J. Neurophysiol. 80, 1439-1455.

47. Steriade M., Contreras D., Curró Dossi R. and Núñez A. (1993) The slow ( $<1 \mathrm{~Hz})$ oscillation in reticular thalamic and thalamocortical neurons: scenario of sleep rhythm generation in interacting thalamic and neocortical networks. J. Neurosci. 13, 3284-3299.

48. Steriade M., Contreras D., Amzica F. and Timofeev I. (1996) Synchronization of fast $(30-40 \mathrm{~Hz})$ spontaneous oscillations in intrathalamic and thalamocortical networks. J. Neurosci. 16, 2788-2808.

49. Steriade M., Contreras D. and Amzica F. (1997) The thalamocortical dialogue during wake, sleep and paroxysmal oscillations. In Thalamus: Experimental and Clinical Aspects (eds Steriade M., Jones E. G. and McCormick D. A.), Vol. II, pp. 213-294. Elsevier, Amsterdam.

50. Steriade M. and Llinás R. (1988) The functional states of the thalamus and the associated neuronal interplay. Physiol. Rev. 68, 649-742.

51. Steriade M., Núñez A. and Amzica F. (1993) A novel slow $(<1 \mathrm{~Hz})$ oscillation of neocortical neurons in vivo: depolarizing and hyperpolarizing components. J. Neurosci. 13, 3252-3265. 
52. Steriade M., Núñez A. and Amzica F. (1993) Intracellular analysis of relations between the slow $(<1 \mathrm{~Hz})$ neocortical oscillation and other sleep rhythms of the electroencephalogram. J. Neurosci. 13, 3266-3283.

53. Steriade M. and Timofeev I. (1997) Short-term plasticity during intrathalamic augmenting responses in decorticated cats. J. Neurosci. 17, 3778-3795.

54. Timofeev I., Grenier F. and Steriade M. (1998) Spike-wave complexes and fast components of cortically generated seizures. IV. Paroxysmal fast runs in cortical and thalamic neurons. J. Neurophysiol. 80, 1495-1513.

55. Valtschanoff J. G., Weinberg R. J., Rustioni A. and Schmidt H. H. H. W. (1995) Colocalization of neuronal nitric oxide synthase with GABA in rat cuneate nucleus. J. Neurocytol. 24, 237-245.

56. Wen C. Y., Chen K. N., Lue J. H., Chan S. A. and Shieh J. Y. (1992) An electron microscopic and morphometric study on the GABA-immunoreactive terminals in the cuneate nucleus of the rat. J. Anat. 18, 3409-3415.

57. Wiberg M. and Blomqvist A. (1984) The projection to the mesencephalon from the dorsal column nuclei. An anatomical study in the cat. Brain Res. 311, $225-244$. 